NASA Technıcal Memorandum 86782

NASA-TM-86782 19860000548

\title{
Evaluation of an Aerodynamic- Load Prediction Method on a STOL Fighter Configuration
}

Joseph Katz

August 1985

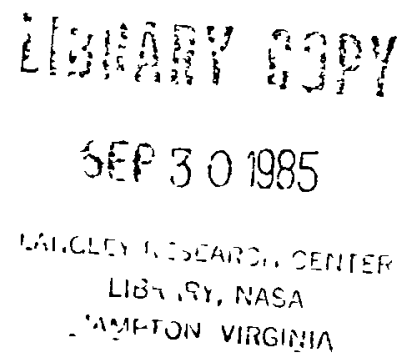


NASA Technıcal Memorandum 86782

\section{Evaluation of an Aerodynamic- Load Prediction Method on a STOL Fighter Configuration}

Joseph Katz, Ames Research Center, Moffett Field, Calıfornıa

August 1985

\section{N/Sก}

Natıonal Aeronautics and

Space Administratıon

Ames Research Center

Moffett Field Calıfornı 94035 


\section{EVALUATION OF AN AERODYNAMIC-LOAD PREDICTIDN METHOD}

ON A STOL FIGHTER CONFIGURATION*

Joseph Katz

Ames Research Center

Moffett Field, CA 94035

*To be published concurrently as NASA TM-86732. 


\section{NOMENCLATURE}

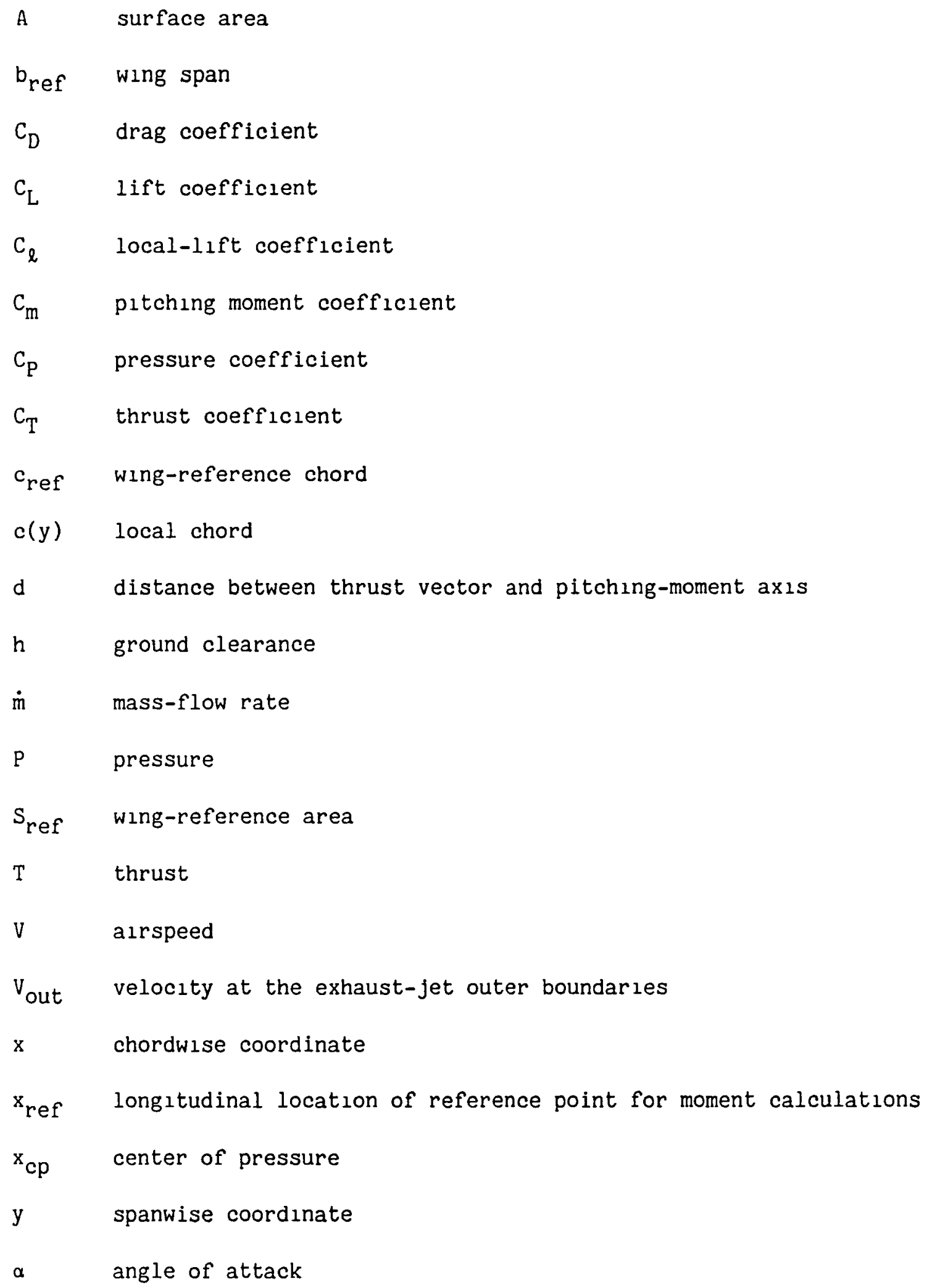


$\rho \quad$ air density

Subscripts

()$_{\mathrm{c}}$ canard

( ) ex exit

()$_{f}$ fuel

( ) in inlet

( ) $)_{\mathrm{T}}$ thrust

()$_{W}$ wing

( ) at geometric angle of attack $=0$

()$_{\infty}$ free-stream condition 


\title{
EVALUATION OF AN AERODYNAMIC-LOAD PREDICTION METHOD
}

\author{
ON A STOL FIGHTER CONFIGURATION
}

Joseph Katz

Ames Research Center

SUMMARY

A three-dimensional panel method was used to compute the aerodynamic loads on a large-scale, powered wind tunnel model. The size of the computation grid was selected such that turnaround times, on present computers, remained satisfactory. With this constraint, the STOL aircraft configuration was modeled and the capabilities and limitations of the prediction method were investigated. After this study, a simllar numerical model was established for a forward-swept-wing alrcraft configuration which is planned for large-scale, low-speed testing. The resulting aerodynamic-load predictions are discussed and will be utilized, together with the future experimental results, to obtain a broader validation of this method as well as to reduce the necessary size of the matrix of the test parameters.

\section{INTRODUCTION}

Accurate prediction of the performance and stability of aircraft and high-speed vehicles by the use of experimental and analytical methods is essential for reliable development of a final configuration. As the initial geometry of the vehicle is established, its aerodynamic characteristics have to be continually evaluated. Wind tunnels take an active role in the development of complex aircraft shapes such as a $\mathrm{V} / \mathrm{STOL}$ (vertical short takeoff and landing) configuration, so that sufficient fullscale details are extracted for an accurate assessment of the vehicle's aerodynamic characteristics. Such a level of detalling is needed to investigate low-speed and high-angle-of-attack conditions, to account for Reynolds number effects, and to evaluate the influence of the smaller geometric details. The procedure is long and costly, and at several stages of development these large or full-scale tests are needed to investigate flight conditions such as landing, takeoff, and transition. Therefore, inclusion of advanced prediction methods into the various phases of a development program has the potential to shorten both model-design and wind tunnel test time. This integration can be accomplished by computing data for the preliminary performance estimation, for the structural design of the model, and for planning the matrix of the test parameters. Part of the results of these computations are in the form of numerical flow visualization. Streamline plots, for example, may provide an explanation for flow features (as in the case of vortex flows) and can point toward possible improvements in a redesign exercise of the model geometry (e.g., in the case of undesirable vortex interactions with lifting surfaces). 
For the low-speed flow regime, aerodynamic load prediction methods already have been developed to the point where turnaround times on today's computers allow an almost-real-time computation. In this report, a validation of one of these methods is presented. In the first part of this investigation, computed data were compared with full-scale wind tunnel results for a V/STOL aircraft configuration. Then, aerodynamic-load predictions were computed for a future V/STOL model, which is now under construction and will be tested in the full-scale wind tunnel. This validation procedure ensures that predicted results are computed without prior knowledge of the measured quantities.

\section{DESCRIPTION OF THE PREDICTION METHOD}

The fluld-dynamics equations involved in solving the pressure field of a complex V/STOL aircraft are basically nonlinear. This nonlinearity is a result of the viscous terms associated with flow separation, vortex flows, propulsion jets, boundary layers, and recirculating flows caused by the interaction of jets and ground. A complete solution of these equations for a real aircraft shape will require an elaborate and time-consuming (ref. 1) computer code (compared with linearized methods). On the other hand, during the past 20 years experience has been gained in solving the linear portions of these fluid dynamic equations (refs. 2-12). These solutions predict the fluid dynamics of inviscid regions which include the lifting properties of nonseparated wings in subsonic or supersonic flows (refs. 4,7,8). An additional advantage of the potential problem emerges from the use of Green's theorem (ref. 9): an integral equation is obtained, which is solved on the body's boundaries only, rather than a solution spanning the whole fluid region. A comprehensive description of the mathematical principles and of the required numerical schemes of these methods are provided in references 7-12. These methods were extended to include more complex singularity elements (refs. 4,12), interaction with viscous boundary-layer solutions, wake rollup relaxations, and jet models (ref. 13). The code VSAERO (refs. 5,13), used in the current investigation, included most of the aforementioned features and was found to be among the most efficient (ref. 6), in terms of computation time.

\section{PANELED WIND TUNNEL MODEL}

Once the computation method was selected, its capability and limitations were investigated via a comparison with experimental results. The full-scale wind tunnel model that was used for this validation is shown in figure 1. Additional geometric details on the model are presented in figure 2 and in references 14-17. The panel model, for this configuration, consisted of 767 panels and computation times were about $60 \mathrm{sec}$ on a CRAY XMP computer, for one run through the program. The flow in the inlet was modeled by specifying an inward, normal velocity of about 1.2 times the free-stream velocity. More complex inlet flows already have been modeled using this method (ref. 13). Because of the large increase in the required number of 
panels and because of the longer computational time, this fine detailing was not used here. The exhausting jets were modeled by enclosing the high-energy jet region with a linearily varying doublet sheet, equivalent to a constant-strength vortex sheet, as shown in figure 3. The strength of this doublet sheet was obtained by prescribing the corresponding inner and outer jet velocities. In the actual tests (refs. 14-16) the jet engine was operating at a constant setting and the thrust coefficient was varled by changing the wind tunnel airspeed. The engine-exhaust total temperature was about $890 \mathrm{~K}$ with an average (inner) velocity of about $457 \mathrm{~m} / \mathrm{sec}$. For a thrust coefficient of unity this will yleld to a velocity ratio $\left(\mathrm{V}_{\mathrm{ex}} / \mathrm{V}_{\infty}\right)$ of almost 11 . To account for the correct amount of vorticity generated at the shear layer, a density correction of 2.9 was applied because of this temperature difference (refs. 14-16) (based on test conditions). Therefore, the velocity ratios used in this report were $3.42,3.70,4.53,5.31$, and 5.90 for the corresponding thrust coefficlents of $0.9,1.0,1.4,1.8$, and 2.0 , accordingly. The outer velocity $\left(V_{\text {out }}\right)$ in figure 3 was matched with the computed external velocity and was close to $\left(V_{\text {out }} / V_{\infty}=1.2\right)$. This model was investigated and used for various applications (refs. 13,18); however, it lacks a detailed simulation of the jet/free-stream entrainment.

To account for the contribution of the jet engines to the aerodynamic forces and moments, the formulation presented in equation (1) is used. Here $\alpha_{\mathrm{T}}$ is the angle between the jet vector and the alrcraft geometrical axis and $d$ is the distance between this vector and the reference point (center of mass) as shown in figure 4.

$$
\begin{aligned}
& \Delta C_{\mathrm{LT}}=\mathrm{C}_{\mathrm{T}} \sin \left(\alpha+\alpha_{\mathrm{T}}\right) \\
& \Delta \mathrm{C}_{\mathrm{DT}}=-\mathrm{C}_{\mathrm{T}} \cos \left(\alpha+\alpha_{\mathrm{T}}\right) \\
& \Delta \mathrm{C}_{\mathrm{mT}}=-\mathrm{C}_{\mathrm{T}} \mathrm{d} / \mathrm{c}_{\text {ref }}
\end{aligned}
$$

Additionally, a distinction has to be made between the "jet" and "pressure" components of the thrust force. From simplified one-dimensional momentum considerations the thrust of an isolated jet engine includes the following terms:

$$
\begin{aligned}
T=\int_{\text {nacelle }} V(\rho V \cdot d A)+ & \int_{\text {nacelle }} P \cdot d A \\
& =\sim\left(\dot{\mathrm{m}}_{\text {in }}+\dot{\mathrm{m}}_{\mathrm{f}}\right) \mathrm{V}_{\text {ex }}-\dot{\mathrm{m}}_{\text {in }} \mathrm{V}_{\text {in }}+\left(\mathrm{P}_{\text {ex }}-\mathrm{P}_{\infty}\right) A_{\text {ex }}-\left(\mathrm{P}_{1 \mathrm{n}}-\mathrm{P}_{\infty}\right) \mathrm{A}_{1 \mathrm{n}}
\end{aligned}
$$

The last two terms, which are a result of the pressure integral, are actually included in the numerical computation. When accounting for the thrust of the jet (e.g., $C_{T}=1$ ), however, all the terms of equation (2) are included. To avoid the inclusion of these terms twice, the results of the pressure integral over the jetengine nacelle, [ ] nacelle, has to be subtracted from the overall pressure integration results, [ ] VSAERO: 


$$
\left[\begin{array}{l}
C_{L} \\
C_{D} \\
c_{m}
\end{array}\right]_{\text {model }}=\left[\begin{array}{l}
C_{L} \\
C_{D} \\
C_{m}
\end{array}\right]_{\text {VSAERO }}-\left[\begin{array}{l}
\Delta C_{L} \\
\Delta C_{D} \\
\Delta C_{m}
\end{array}\right]_{\text {nacelle }}+\left[\begin{array}{l}
\Delta C_{L T} \\
\Delta C_{D T} \\
\Delta C_{m T}
\end{array}\right]
$$

\section{COMPARISONS WITH EXPERIMENTS}

Evaluation and validation of the prediction method was obtained by comparing the computed results with the experimental measurements of references 15 and 16 . In these wind tunnel tests, apart from the integral forces, pressure data were also recorded along the cross sections ( $A-A, B-B, C-C$, and $D-D$ ) shown in figure 2. These data, together with the computed pressure values, are presented in figures 5-8 without power effect and in figures 9-12 with the effect of the propulsion jets. The pressures on both upper and lower surfaces of the canard are presented in figure 5. At the smaller angle of attack there is a good agreement between the measured and computed data, excluding a limited area at the upper surface of the leading edge (LE). In this region the potential predictions overestimate the suction peaks, which are limited by the viscous effects. At the higher angles of attack, a LE vortex is developed at the canard's upper surface and a larger deviation in the pressure distribution is observed. Since the canard sweep-back angle is only $45^{\circ}$, the resulting vortex effect is small (ref. 19), and in spite of this discrepancy the overall lift is approximated quite well. This behavior is less pronounced in the case of the wing that has an aft-sweep of $40^{\circ}$. Therefore, for the inboard section ( $B-B$ in $f 1 g .2$ ), the measured results in figure 6 fall closer to each other than for the wing's outboard section C-C ( $f 1 g .7$ ), where the influence of LE separation (refs. 14-16) caused a small offset between the experiment and the prediction. The comparison of the pressure data, in the case of the strake (fig. 8) exhibits similar behavior. At the larger angles of attack, traces of LE separation are again evident and inclusion of LE vortex models (e.g., refs. 20,21) might improve the predicted pressure distributions.

The effect of power $\left(C_{\mathrm{T}}=1.4\right)$ on the canard's pressure distribution is small, with a limited increase of the vortex lift at the front section of the alrfoll (fig. 9). At the wing's inboard ( $f 1 g .10$ ) and outboard sections ( $f 1 g .11$ ), the prediction is satisfactory and again a limited increase in the lift of the section caused by power is detected. Also, in the case of the strake ( $f 1 g .12$ ), the changes in both measured and calculated pressures are smaller; therefore, a good prediction of the lift-curve slope in the nonseparated region is obtained (fig. 13). When computing the "power-on" effects, a jet angle ( $a_{T}$ in eq. (1) and in fig. 4 ) of $8.5^{\circ}$ was assumed. This inclination was a result of the exhaust nozzle inner geometry as shown in references 14 and 16 . The experimental data on figure 13 indicate that wing stall is delayed to higher angles of attack for the power-on conditions. Such a nonlinear feature cannot be predicted with this type of computation. 
The V/STOL configuration of figures 1 and 2 has already been analyzed with the current prediction code (VSAERO in ref. 13) and with other panel methods (refs. 22,23). The present panel model, however, has a modified geometry which was a result of a comparison with the actual wind tunnel model, and thereby included a vertical fin as well. Also, the jet-wake and jet-flap modeling (fig. 3 ) were changed relative to the model used in reference 13 . The results of the pressure distribution of figures 5-12 are close to the computed pressures of reference 13 , but their lift curve had slightly lower results than those shown in figure 13 . For comparison, computations for this configuration by other panel methods are also presented in figure 13. The lift values of references 22 and 23 have slightly lower values and smaller slopes. The computations of reference 23 , using the model of reference 12, included some surface pressure computations and had no power effects.

Drag polars and pitching moments for this model are shown in figure 14 and in figure 15. The predicted drag coefficient consists of the lift-induced terms only, and therefore had smaller values. The skin friction on the model was separately computed with the current prediction method and was found to be about $C_{D o}=0.01$. However, this value is small compared with the difference (of about 0.05) implied by figure 14, and is a result of numerous local flow separations around the nacelle and near the wing and canard fuselage junctures. The pitching-moment predictions were sensitive to wake iterations; therefore, instead of a solid line, a predicted range is presented. However, predicted center of pressure was reasonable from the engineering point of view ( $x_{c p} / c_{\text {ref }}=-0.10$ to 0.22 ) compared $w 1$ th the measured value 0.20-0.23 (unstable), reported in reference 14 .

\section{AERODYNAMIC LOAD PREDICTIONS FOR A FORWARD-SWEPT-WING AIRCRAFT MODEL}

Inclusion of advanced aerodynamic prediction techniques into generic test programs can result in improvements in model shape and structural design, improvements in model mounting in the wind tunnel, and in establishing the test program. In this latter case the identification of more dominant test parameters (e.g., canard locations, flap setting, etc.) can contribute to the preparation of a more efficient test matrix. An example of a set of predictions has been chosen for a STOL-fighter configuration scheduled for future large-scale testing. The results of these predictions, apart from the immediate benefits, when combined with future wind tunnel results will provide additional evaluation of this prediction method.

The model, whose geometry is presented in figures 16 and 17, is planned to evaluate the low-speed aerodynamics of forward-swept wings utilizing vectored jets and the control of high-angle-of-attack flow separation by various blowing techniques. The nacelle, tall, canard, and upper portions of the fuselage of this design are similar to those of the model presented in figures 1 and 2 . The strake, however, was removed and the fuselage sides, tail, and wing were redesigned. The reference chord was based on the chord of the wing, without the aft-swept fairing, at the nacelle root section. The reference point $\left(x_{\text {ref }}\right)$ for the moments calculations was left at the same point where it was for the model shown in figures 1 
and 2. For the computations three different canard positions were considered. The model of figure 1 also had this geometric variable, but the results of figures 5-15 included computations for the canard's aft-position only. These three canard locations are on the same horizontal plane with $0.30 \mathrm{~m}$ spacing, as shown by figure 17 . Portions of the planned test include component rotations and dislocations (canards flaps, etc.) that fall within the aerodynamically linear region. At this region, therefore, fewer test points will be required and additional test time can be utilized to investigate complex flow regimes (e.g., separated flows and jet interaction).

The predictions for the lift and drag are presented in figures 18 and 19 for the forward-swept-wing fighter configuration with power effects. Since there might be a small difference in the zero angle of attack between the panel and the actual model, at least two test points will be required to validate the predicted lift slope and $C_{L_{0}}$. The computed induced drag is presented in figure 19. The zero-lift drag coefficient $C_{D o}$ can be roughly estimated from the validation tests shown in figure 14. A separate diagram for the pitching moment is not presented here since the produced curve was sensitive to the wake-iteration routine in the code. The center of pressure $x_{c p}$ for $C_{T}=0$, was estimated to be at the range of $x_{c p} / c_{\text {ref }}=0.00-0.12$, which is ahead of the reference point shown in figure 17 .

The canard in the planned experiment could be mounted at three different positions, as shown by the insert in figure 20. This figure also shows the effect of this parameter on the pitching moment. A forward-canard position will result in higher positive-pitching moments because of its larger distance from the axis of reference $\left(x_{\text {ref }}\right)$. This reference point was positioned near the LE of the wing root (fig. 17), and without the canard the center of pressure was about $0.3 \times c_{\text {ref }}$ behind this axis. With the canard, however, this point was too far aft for static longitudinal stability. Even so, the canard at any position was capable of controlling the configuration. The spanwise loading of the wing, with and without the canard, is shown in figure 21. Because of the aft sweep at the wing's root region, the loading is increasing toward the outer sections. On the forward-swept portion of the wing, however, this behavior is reversed, and the increased loading is directed toward the juncture. Consequently, for the "wing-only" configuration, the peak load is found close to the juncture. When adding a canard in front of the wing its vortex wake induces an upwash at the wing's outboard region and a downwash at the inner sections. This effect is stronger for the canard-aft position. The spanwise loading on the canard, on the other hand, increases as it is shifted backward because of the increased upwash of the wing ( $f 1 g .22$ ). For the computations presented in figures 20-22 the canard was paneled smoothly into the nacelle for the canard-aft position only. For the other canard locations, small gaps in the model geometry (between the canard and the nacelle) were left open to simplify the calculation. The effect of these openings on the panel model were minimized by the small panel sizes near the canard's root. This feature of this prediction method of not being sensitive to small discontinuities in the paneled surface, is important since during wind tunnel testing reasonable predictions can be obtained for unplanned configuration changes. 
The computation of aircraft $11 \mathrm{ft}$ with ground proximity, without propulsion effects for a landing configuration is presented in figure 23. Because of the angle of incidence of the configuration and because of the forward sweep of the wing, in this case, the lifting surfaces lie high above the ground. This effect is more noticeable for the canard and for the forward-swept portion of the wing; therefore, ground proximity has only a small effect on the lift. However, for a landing V/STOL aircraft configuration, the flow field caused by propulsion jets and resulting recirculations is more complex and the simplified model used here is not adequate. Flow visualizations and velocity surveys are also easily computed with this prediction method. These visualizations are usually important during wind tunnel testing, so that a correct physical interpretation of the measured results can be obtained. Such numerical flow visualizations were prepared before the wind tunnel test and are presented in figures 24 and 25 . Here a vector representation of simulated air velocity and directions, for the $C_{T}=0$ condition, are described at the nacelle midsection (fig. 24). The wake of the aircraft is shown in figure 25 . The proximity between the canard's wake and the wing explains the strong interaction presented in figure 21. The short turnaround times required by the panel method to generate such numerical flow visualizations allow real-time display of such results that can be examined while the wind tunnel is running.

\section{CONCLUDING REMARKS}

The panel method that was used here is based on potential flow with modeling of some of the viscous effects. Thereby, when strong viscous regimes exist in the physical case, their results are not accurate everywhere, especially the results taken close to sharp edges of nacelles and wings' leading edges, near jets and nozzles, and when shifting the canard back and forth. However, integral results for lift and drag, and even pressures, away from these troubled areas and junctures were found to be valid. Thus, during wind tunnel testing, rapid and reasonable predictions can be provided for the effects of unplanned component shifting and rotation, even when some slots in the panel model are left open. Furthermore, since such simplified methods (compared to Navier-Stokes solutions) have the potential for being faster and more economical, their performance upgrading has to be considered. Future refinement of this method should concentrate on including interactive graphic routines so that changes in model geometry and investigation of the computed output will be accelerated. Inclusion of a more detalled simulation of wake motion, perhaps by developing a time-stepping version of this code, would allow the modeling of LE separation and thereby extend the prediction range up to higher angles of attack. Also, a more detailed modeling of inlet flows and exhaust jets, with and without ground effect, is needed. 


\section{REFERENCES}

1. Chapman, D. R.: Computatıonal Aerodynamics Development and Outlook. AIAA J., vol. 17 , no. 12,1979 , pp. 1293-1313.

2. Kraus, W.: Panel Methods in Aerodynamics. Numerical Methods in Fluid Dynamics. H. J. Wirz and J. J. Smolderen, eds., McGraw-Hill, 1978, pp. 237-297.

3. Rubbert, P. E.; and Saaris, G. R.: A General Three-Dimensional Potential-Flow Method Applied to V/STOL Aerodynamics. SAE Paper no. 68-0304, 1968.

4. Carmichael, R. L.; and Erıcson, L. L.: PAN AIR--A Higher Order Panel Method for Predicting Subsonic or Supersonic Linear Potential Flows About Arbitrary Configurations. AIAA Paper 81-1255, June 1981.

5. Maskew, B.: Program VSAERO, A Computer Program for Calculating the Nonlinear Aerodynamic Characteristics of Arbitrary Configurations. NASA CR-166476, 1982 .

6. Margason, R. J.; KJelgaard, S. 0.; Sellers, W. L. III; Morr 1s, C. E. K. Jr.; Walkey, K. B.; and Shields, E. W.: Subsonic Panel Methods--A Comparison of Several Production Codes. AIAA Paper 85-0280, Jan. 1985.

7. Woodward, F. A.: Analysis and Design of Wing-Body Combinations at Subsonic and Supersonic Speeds. J. of Aircraft, vol. 5, no. 6, 1968, pp. 528-534.

8. Hess, J. L.: Calculation of Potential Flow About Arbitrary Three-Dimensional Lifting Bodies. Einal Technical Report MDC J5679-01, McDonnell Douglas, Long Beach, Calıfornia, Oct. 1972.

9. Hess, J. L.; and Smith, A. M. 0.: Calculation of Potential Flow About Arbitrary Bodies. Progress in Aeronautical Sciences, vol. 8, Pergamon Press, 1967, pp. 1-138.

10. Woodward, F. A.: An Improved Method for the Aerodynamic Analysis of Wing-BodyTail Configurations in Subsonic and Supersonic Flow. NASA CR-2228, May 1973.

11. Morino, L.: A General Theory of Unsteady Compressible Potential Aerodynamics. NASA CR-2464, 1974.

12. Johnson, F. T.: A General Panel Method for the Analysis and Design of Arb1trary Configurations in Incompressible Flows. NASA CR-3079, May 1980.

13. Maskew, B.; Strash, D.; Nathman, J.; and Dvorak, F. A.: Investıgation to Advance Prediction Techniques of the Low-Speed Aerodynamics of V/STOL Aircraft. NASA CR-166479, 1983. 
14. Falarskı, M. D.; Whitten, P. D.; and Harris, M. J.: Aerodynamic Characteristics of a Large-Scale Model of a Highly Maneuverable Supersonic V/STOL Fighter: STOL Configuration. AIAA Paper 80-0234, Jan. 1980.

15. Howell, G. A.; Crosthwait, E. L.; and Witte, M. C.: Evaluation of Pressure and Thermal Data From a Wind Tunnel Test of a Large-Scale, Powered, STOL Fighter Model. NASA CR-166170, June 1981.

16. Falarski, M. D.; and Dudley, M. R.: Analysis of Data From a Wind Tunnel Investigation of a Large-Scale Model of a Highly Maneuverable Supersonic V/STOL Fighter: STOL Configuration. AIAA Paper 81-2620, Dec. 1981.

17. Howell, G. A.: Test Results of Chordwise and Spanwise Blowing for Low-Speed Lift Augmentation. Proc. of 13th ICAS Conf., vol. 2, Aug. 1982, pp. $1222-1234$.

18. Shollenberger, C. A.: Three-Dimensional Wing/Jet Interaction Analysis Including Jet Distortion Influences. J. of Aircraft, vol. 12, no. 9, 1975, pp. $706-713$.

19. Polhamus, E. C.: Predictions for Vortex Lift Characteristics by Leading Edge Suction Analogy. J. of Aircraft, vol. 8, 1971, pp. 193-199.

20. Katz, J.: Lateral Aerodynamics of Delta Wings With Leading Edge Separation. AIAA J., vol. 22, no. 3, 1984, pp. 323-328.

21. Levin, D.; and Katz, J.: Vortex-Lattice Method for the Calculation of the Nonsteady Separated Flow Over Delta Wings. J. of Alrcraft, vol. 18, no. 12, 1981, pp. 1031-1037.

22. Beatty, T. D.; and Worthey, M. K.: Advanced Prediction Technique for the Low Speed Aerodynamics of V/STOL Aircraft, Vol. I. NASA CR-166442, Mar. 1983.

23. Bhateley, I. C.; Howell, G. A.; and Mann, H. W.: Development of V/STOL Methodology Based on a Higher Order Panel Method. NASA CR-166491, Feb. 1983. 


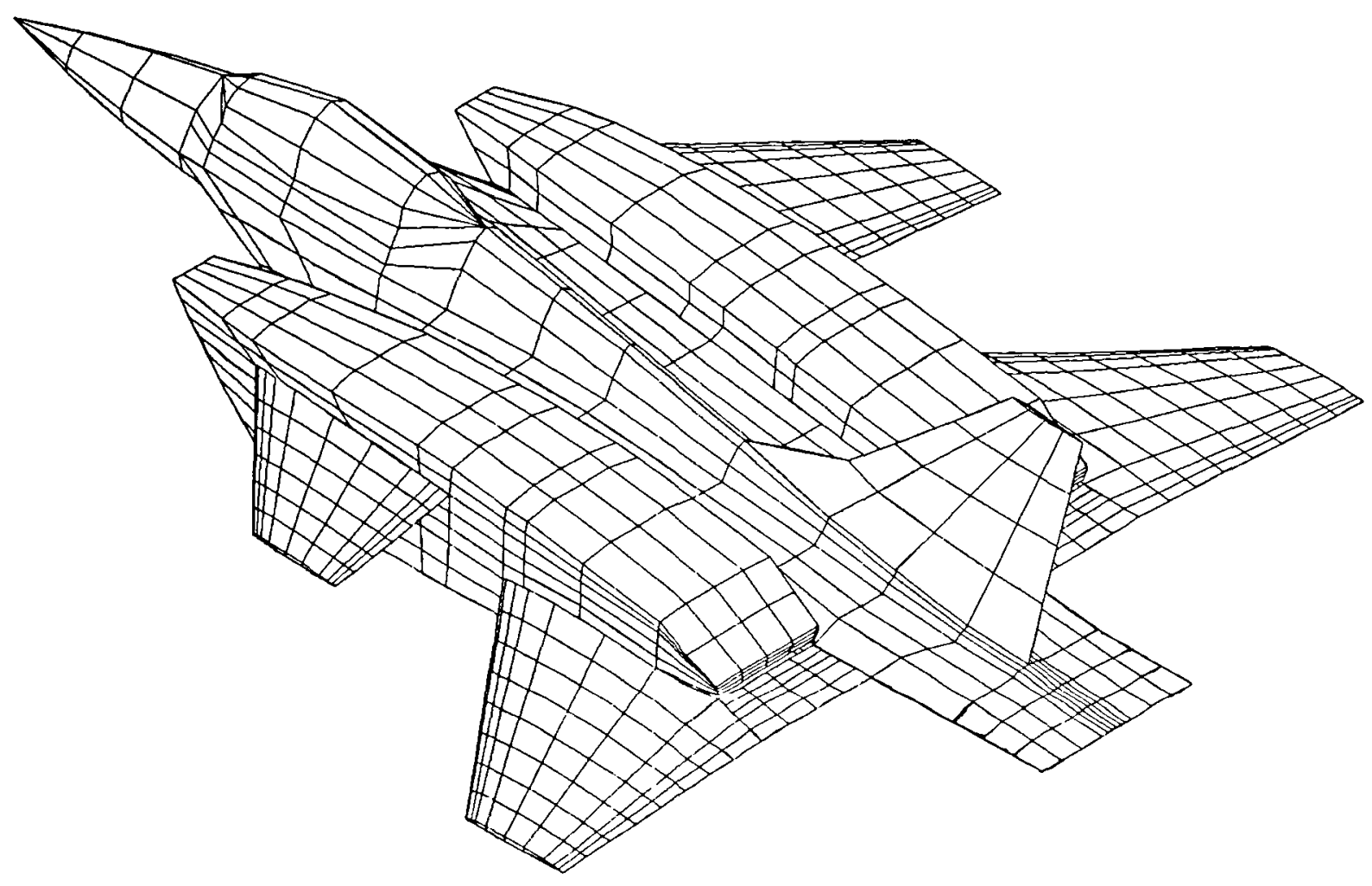

Figure 1.- Panel model of a V/STOL a1rcraft configuration. 


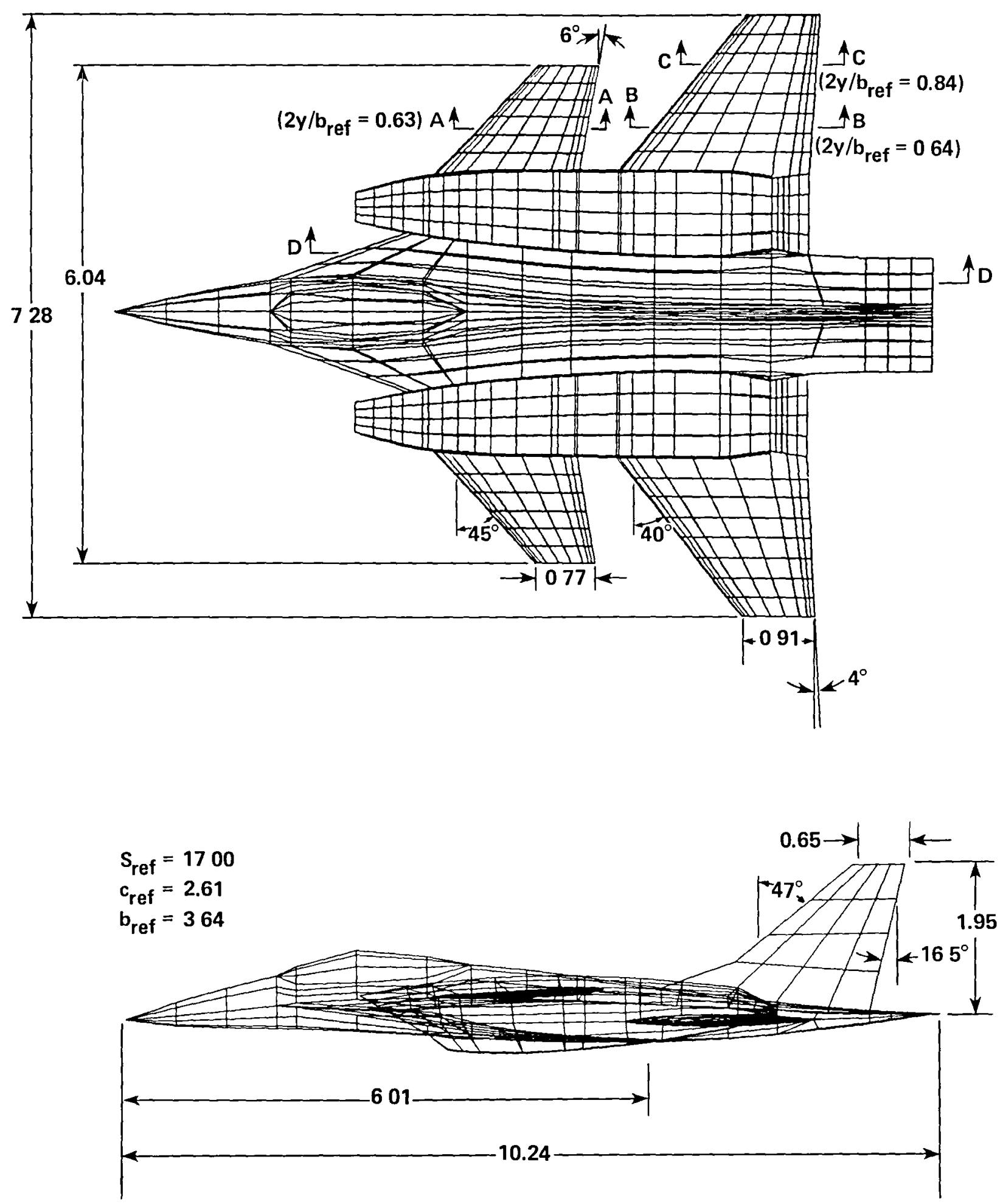

Figure 2.- Geometrical detalls of the V/STOL alrcraft configuration. 


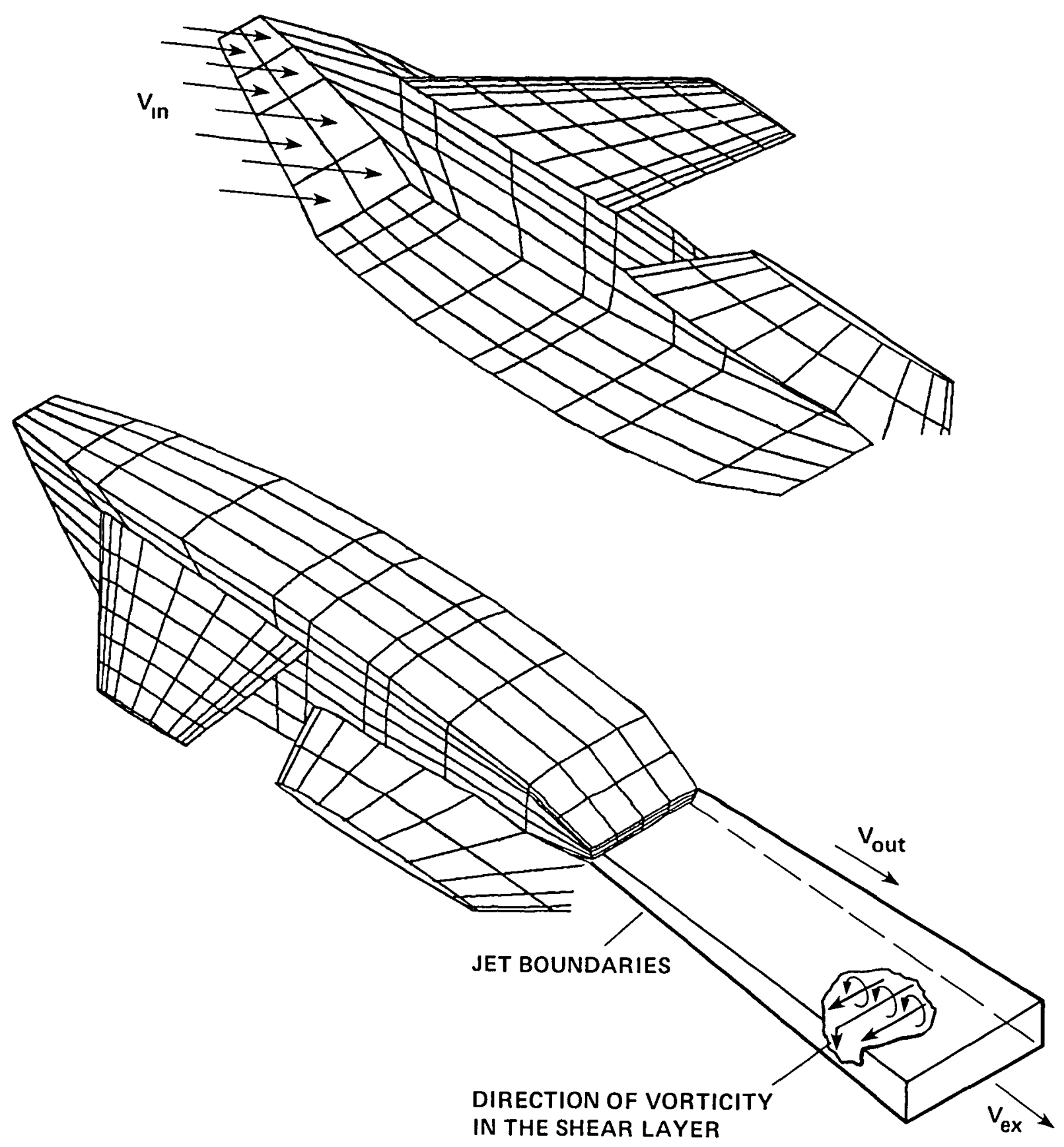

Figure 3.- Models for the inlet and jet flows. 


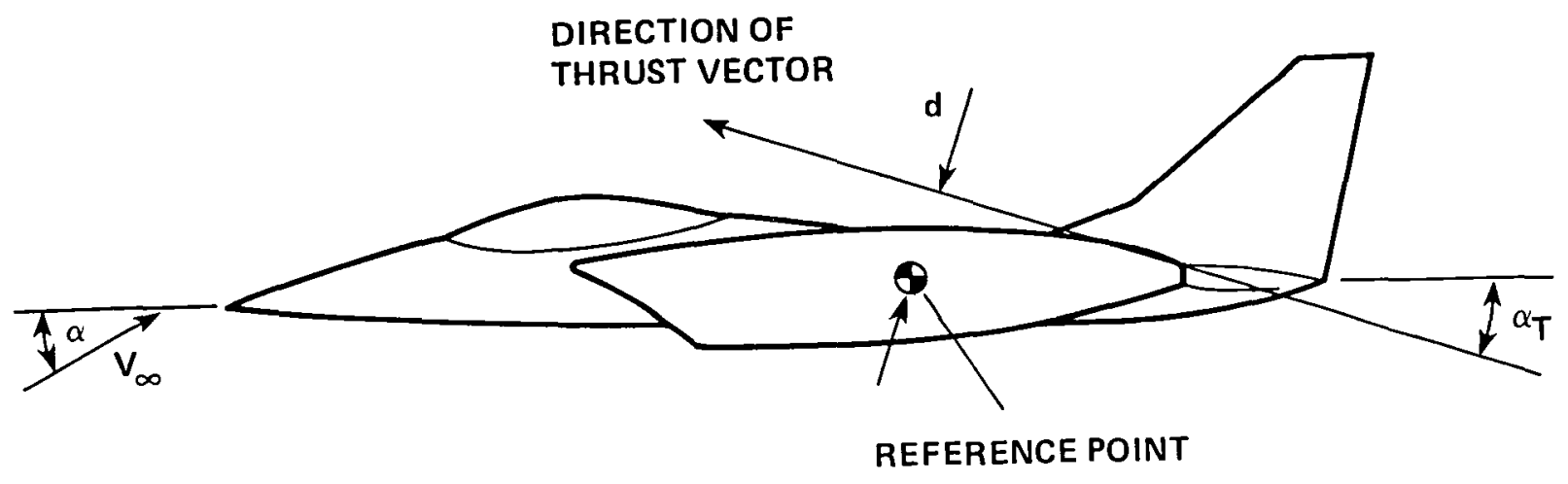

Figure 4.- Thrust vector and model geometries.

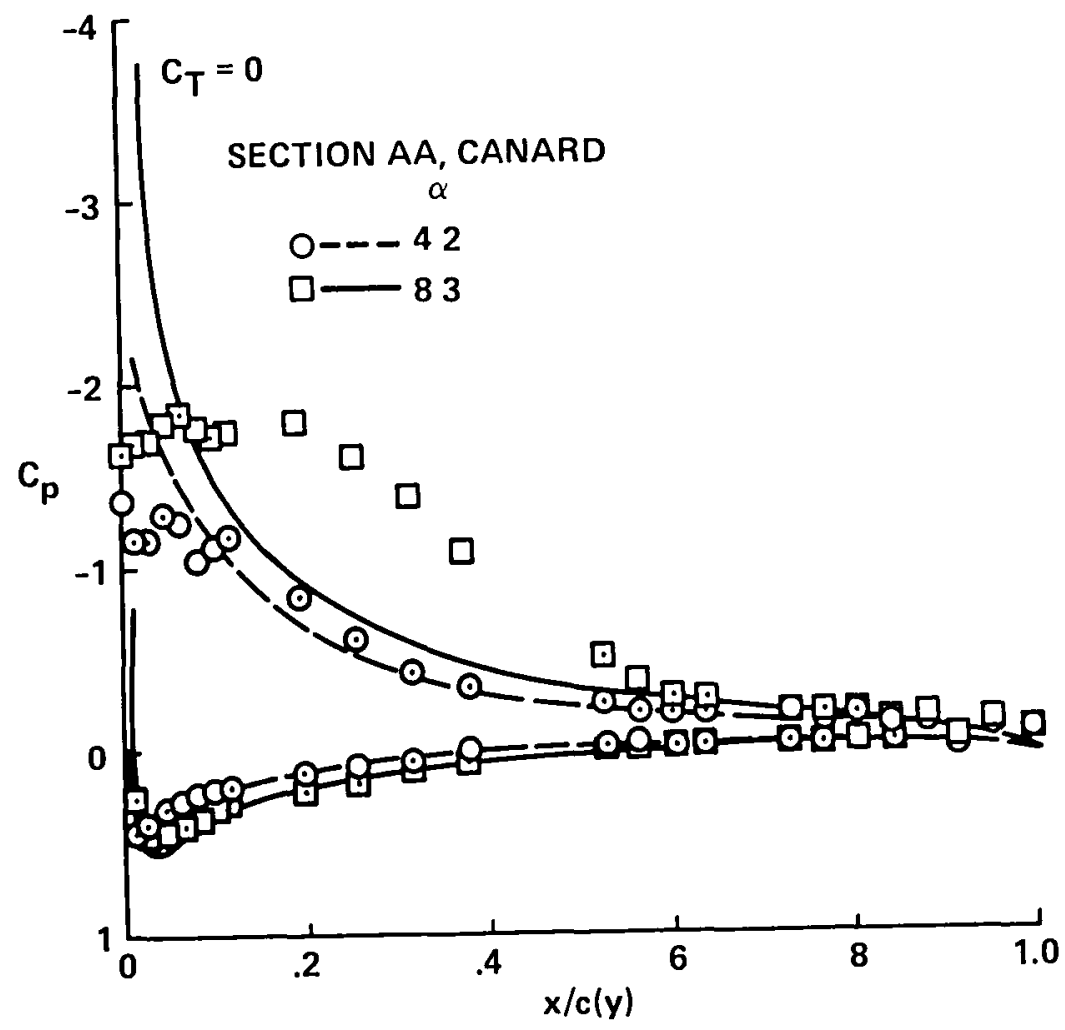

Figure 5.- Pressure distribution on canard. 


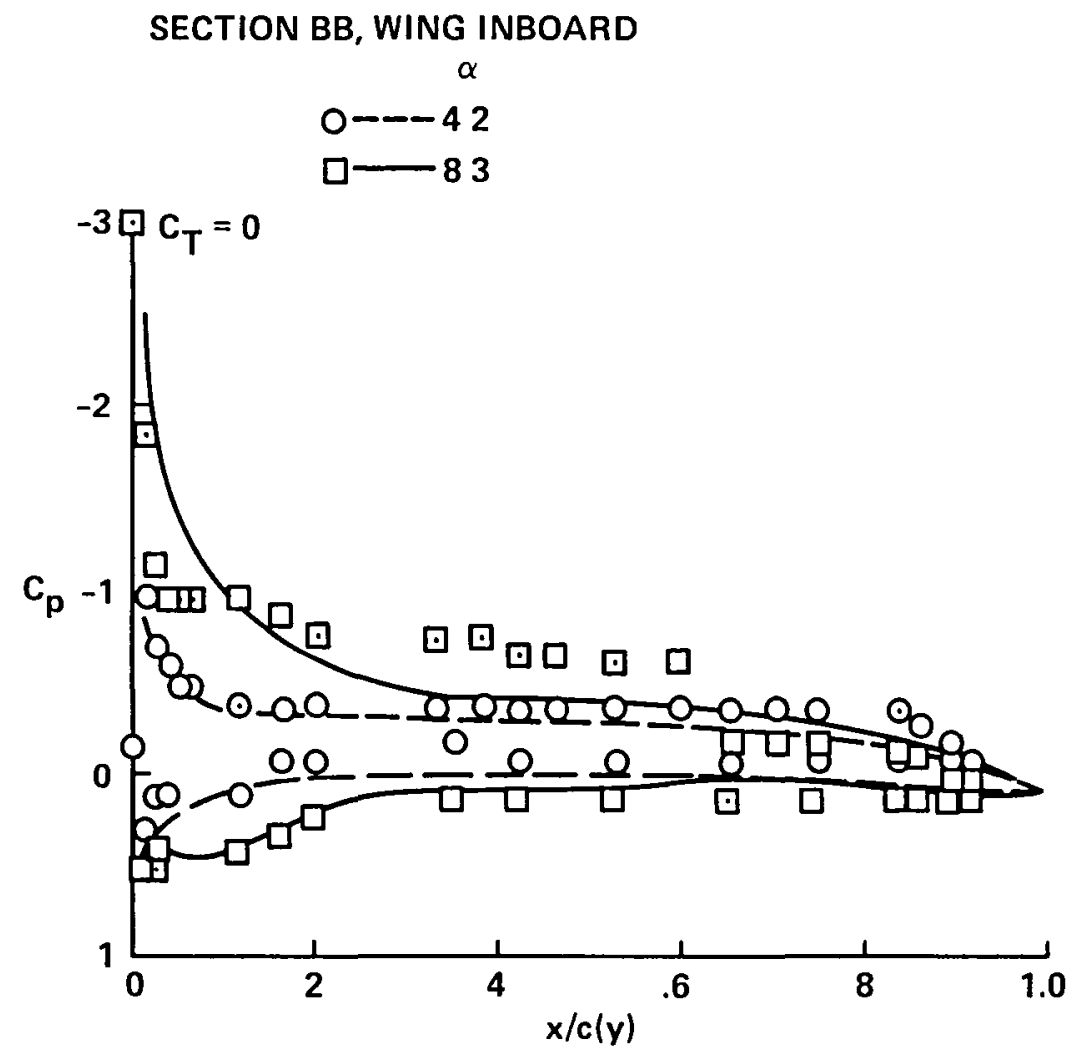

Figure 6.- Pressure distribution on wing (inboard). 


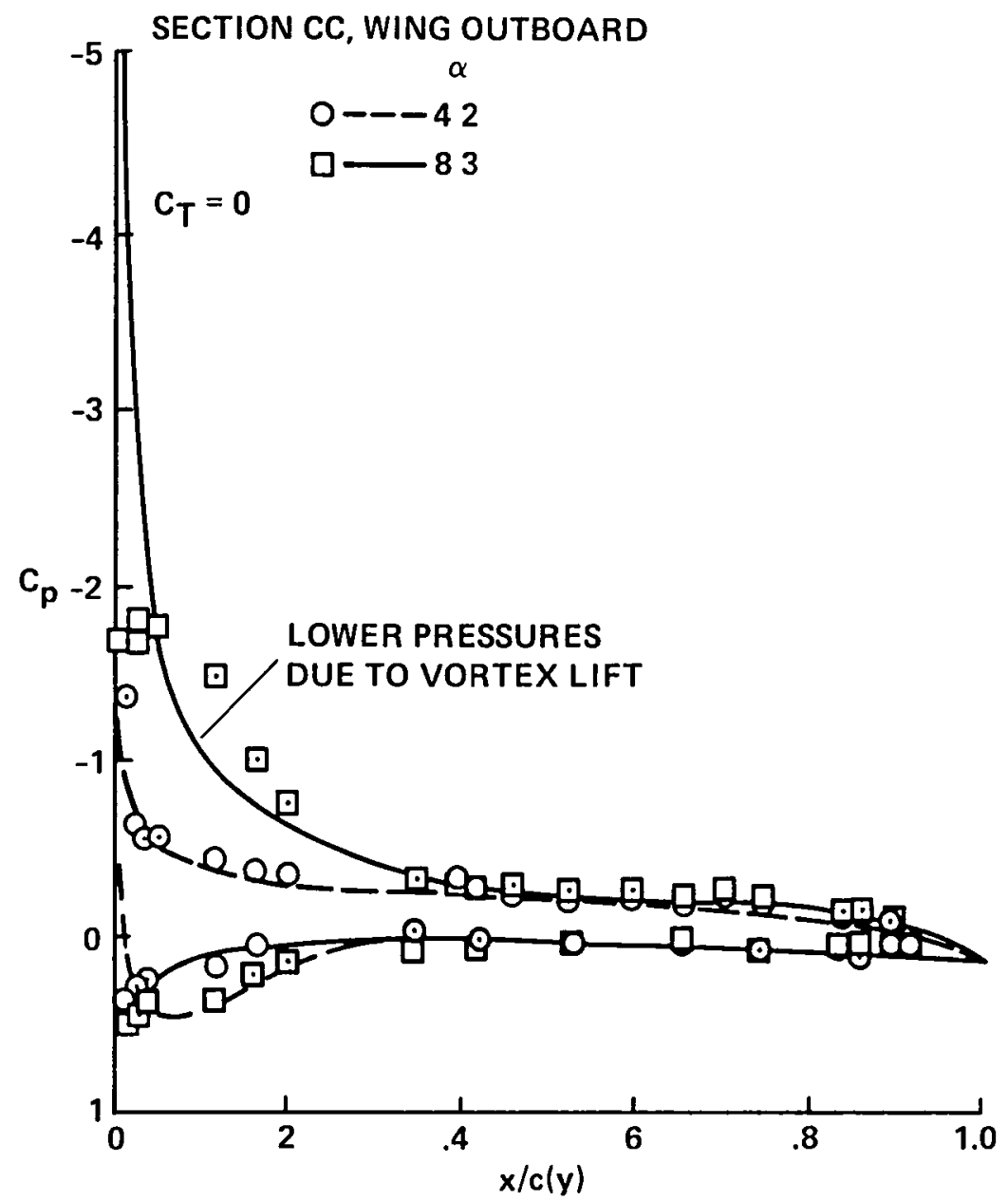

Figure 7.- Pressure distribution on wing (outboard). 


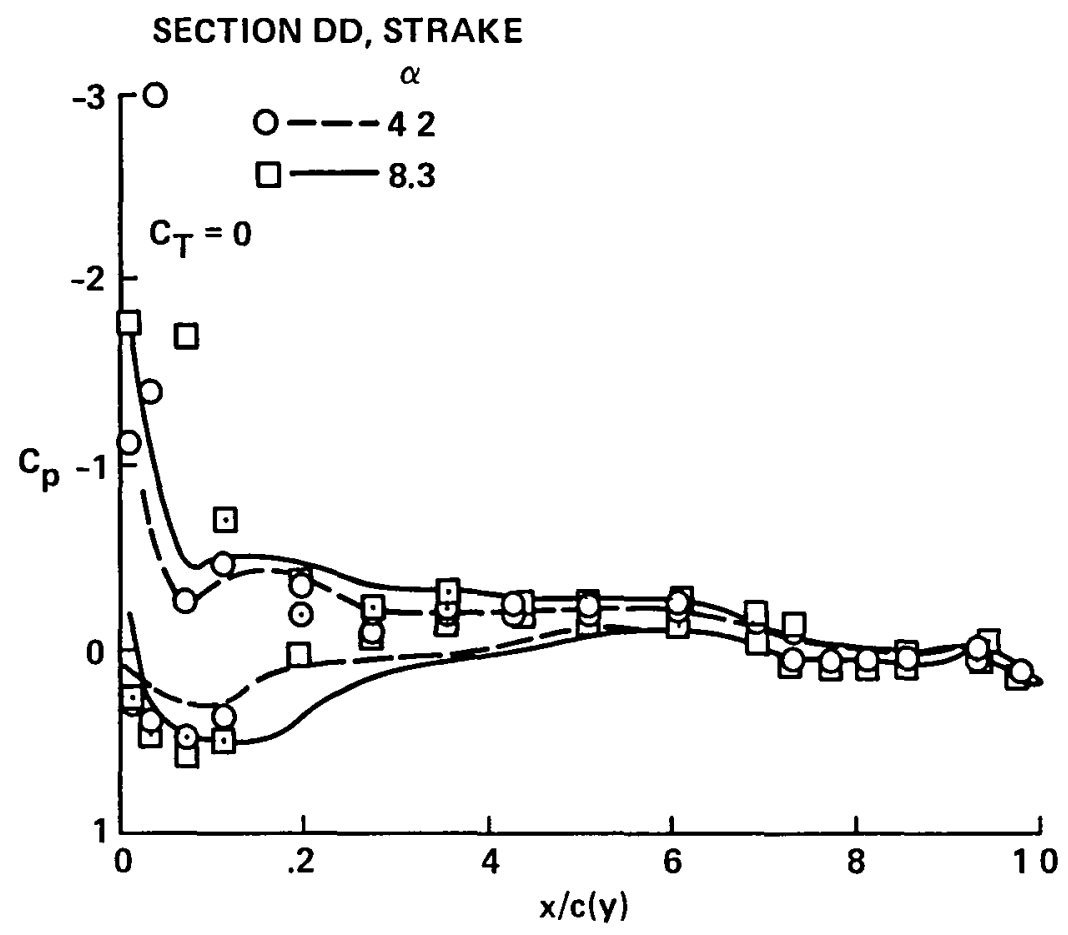

Figure 8.- Pressure distribution on strake.

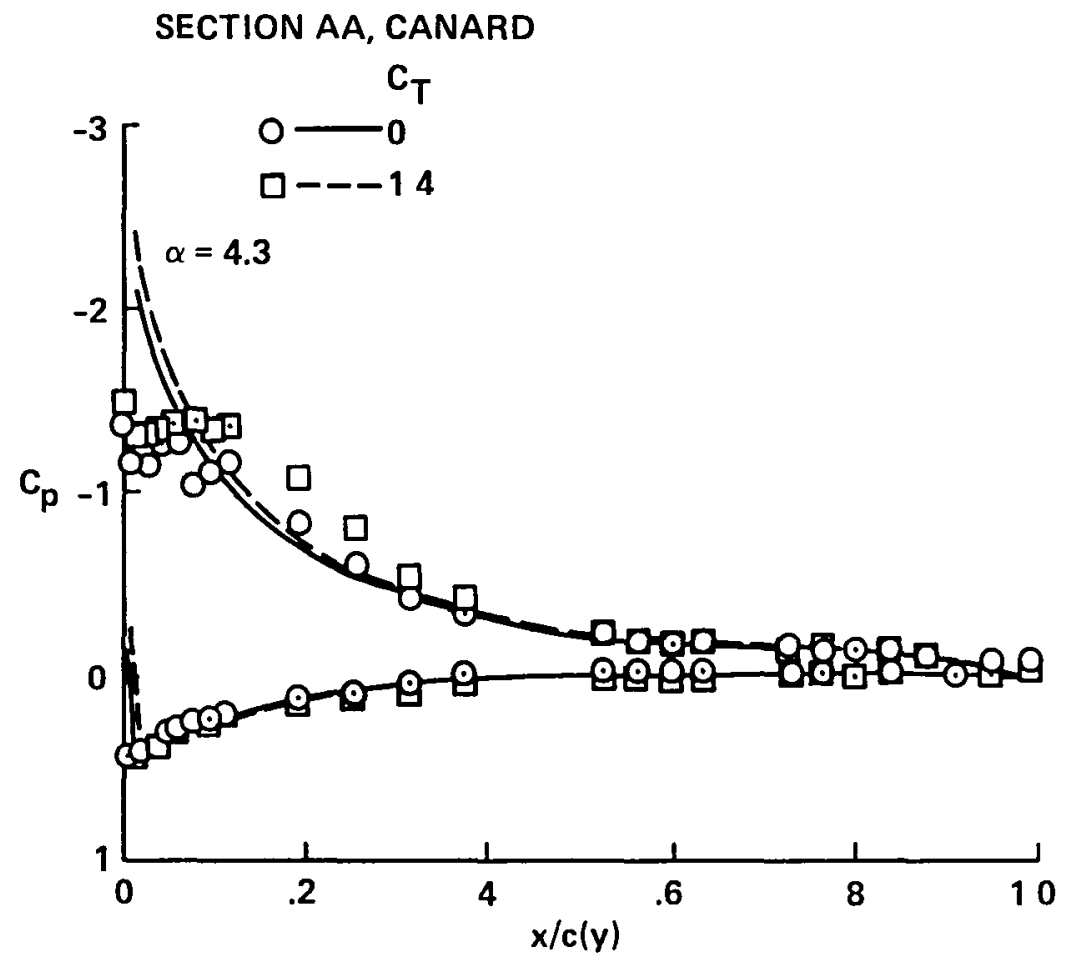

Figure 9.- Effect of power on canard-pressure distribution. 


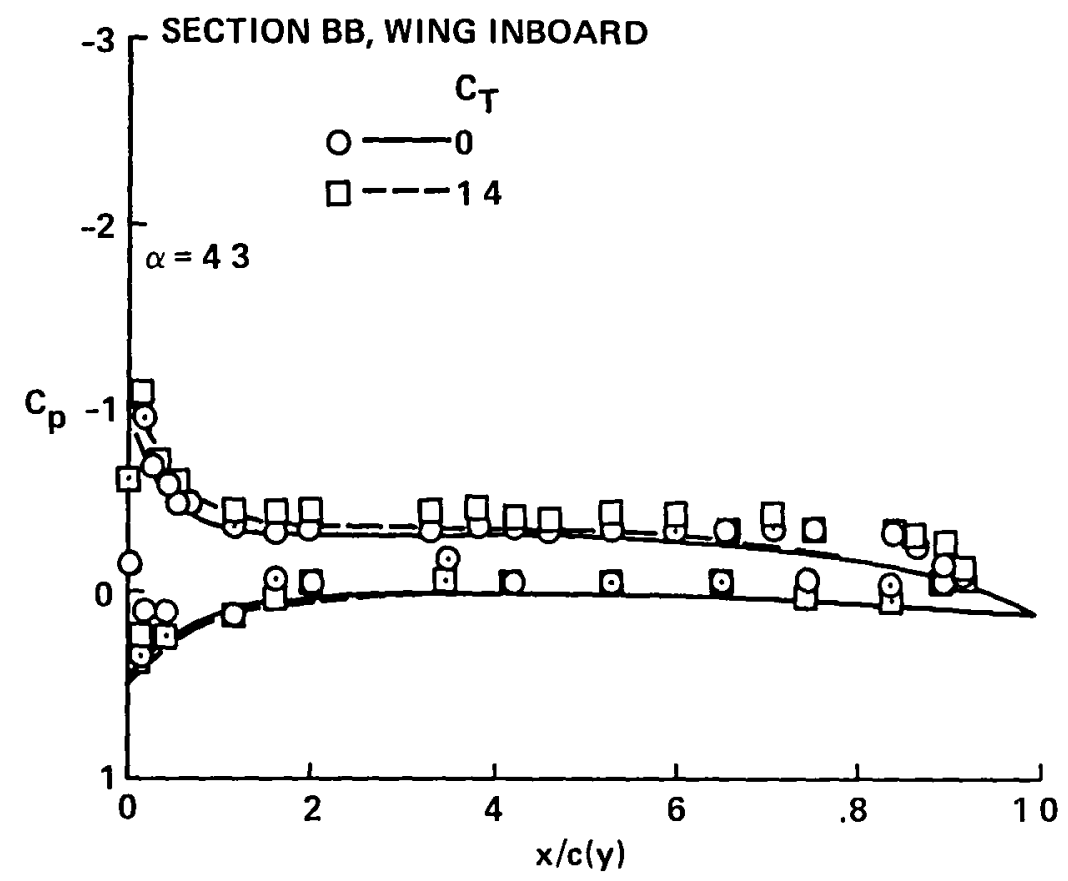

Figure 10.- Effect of power on wing-inboard pressures.

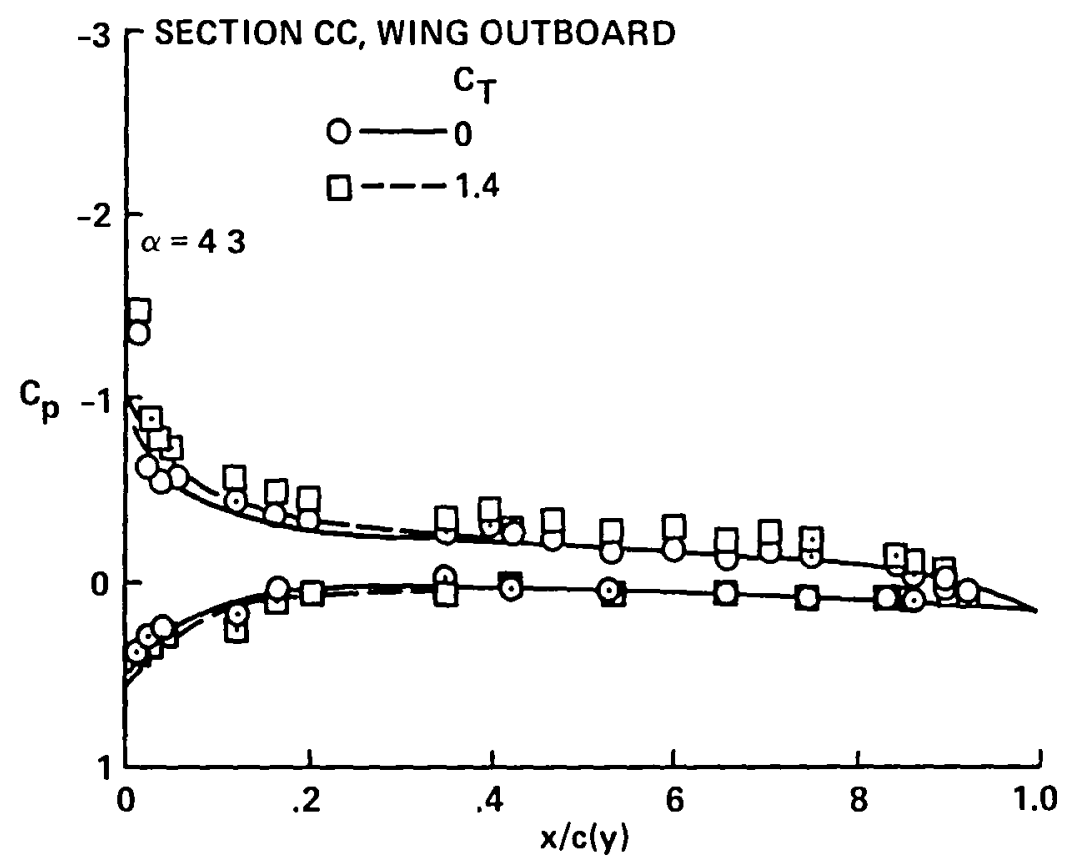

Figure 11.- Effect of power on wing-outboard pressures. 


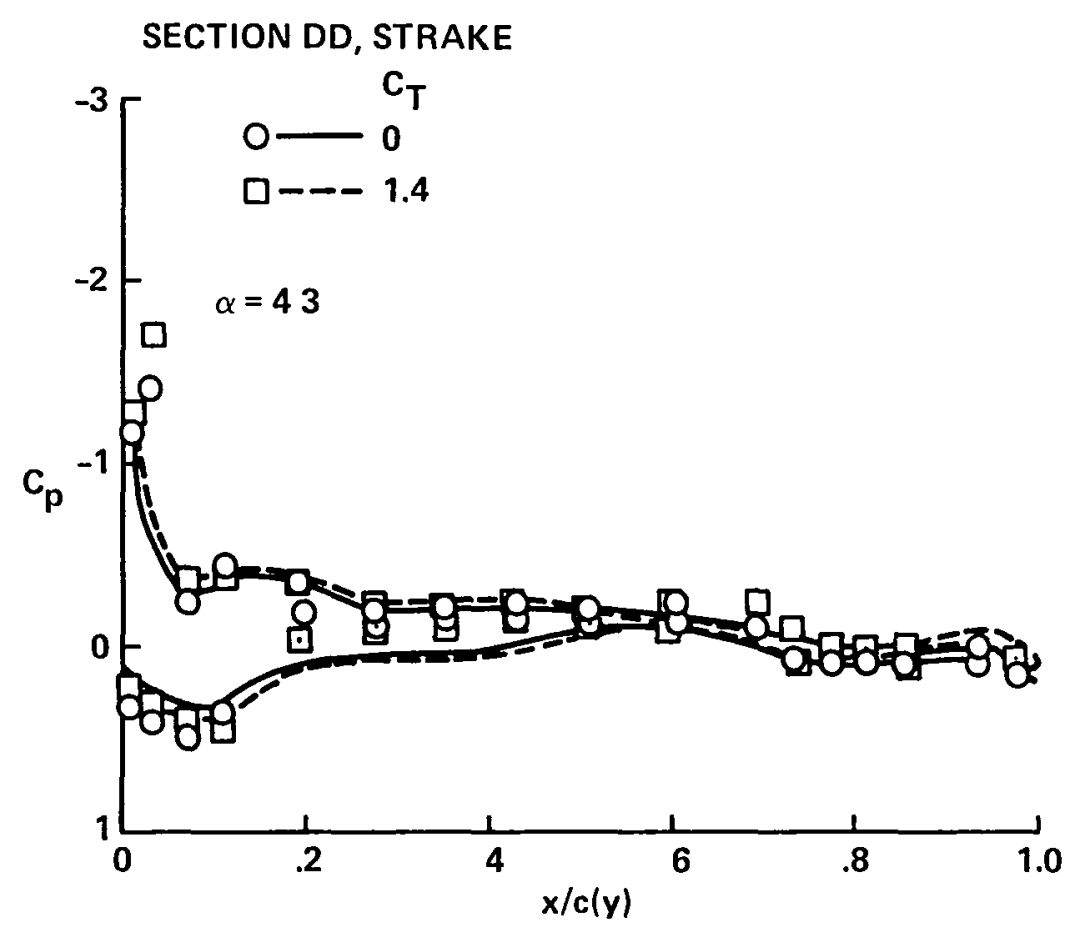

Figure 12.- Effect of power on strake-pressure distribution. 


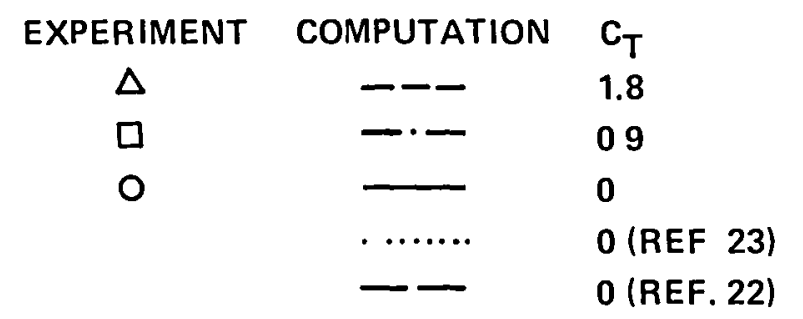

$\Delta$

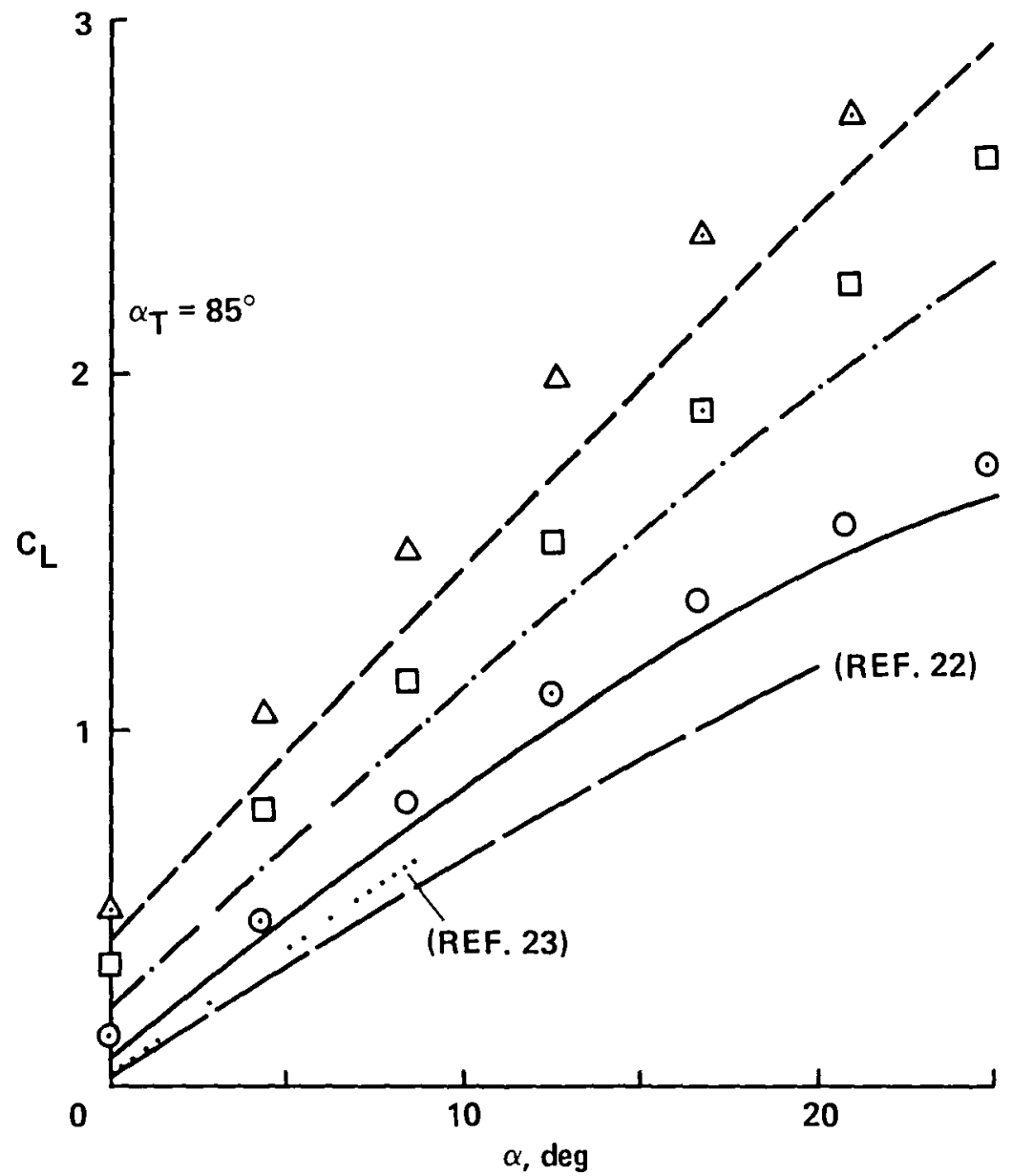

Figure 13.- Lift coefficients for the V/STOL fighter model. 


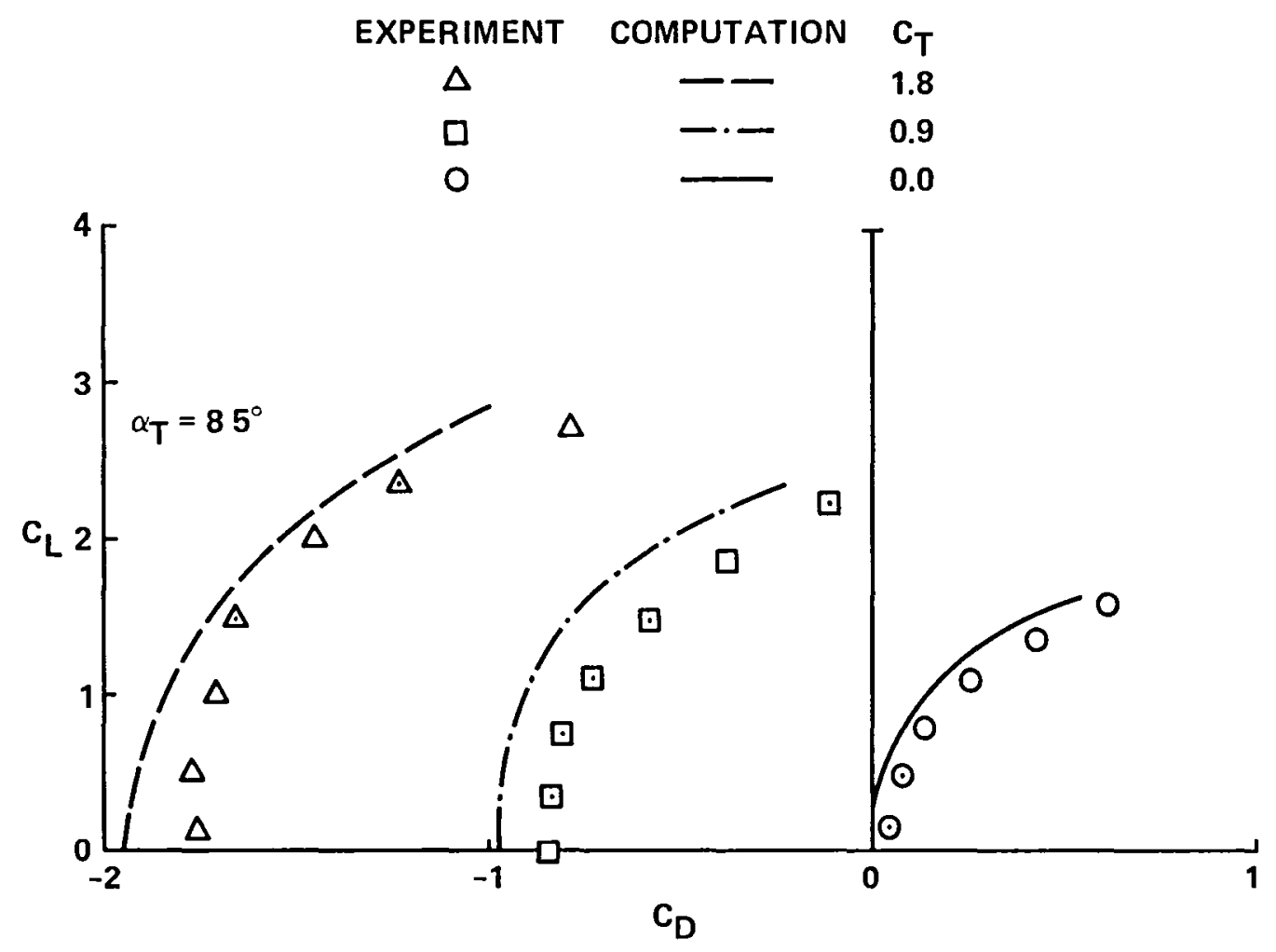

Figure 14.- Lift/drag polars for the V/STOL fighter model. 

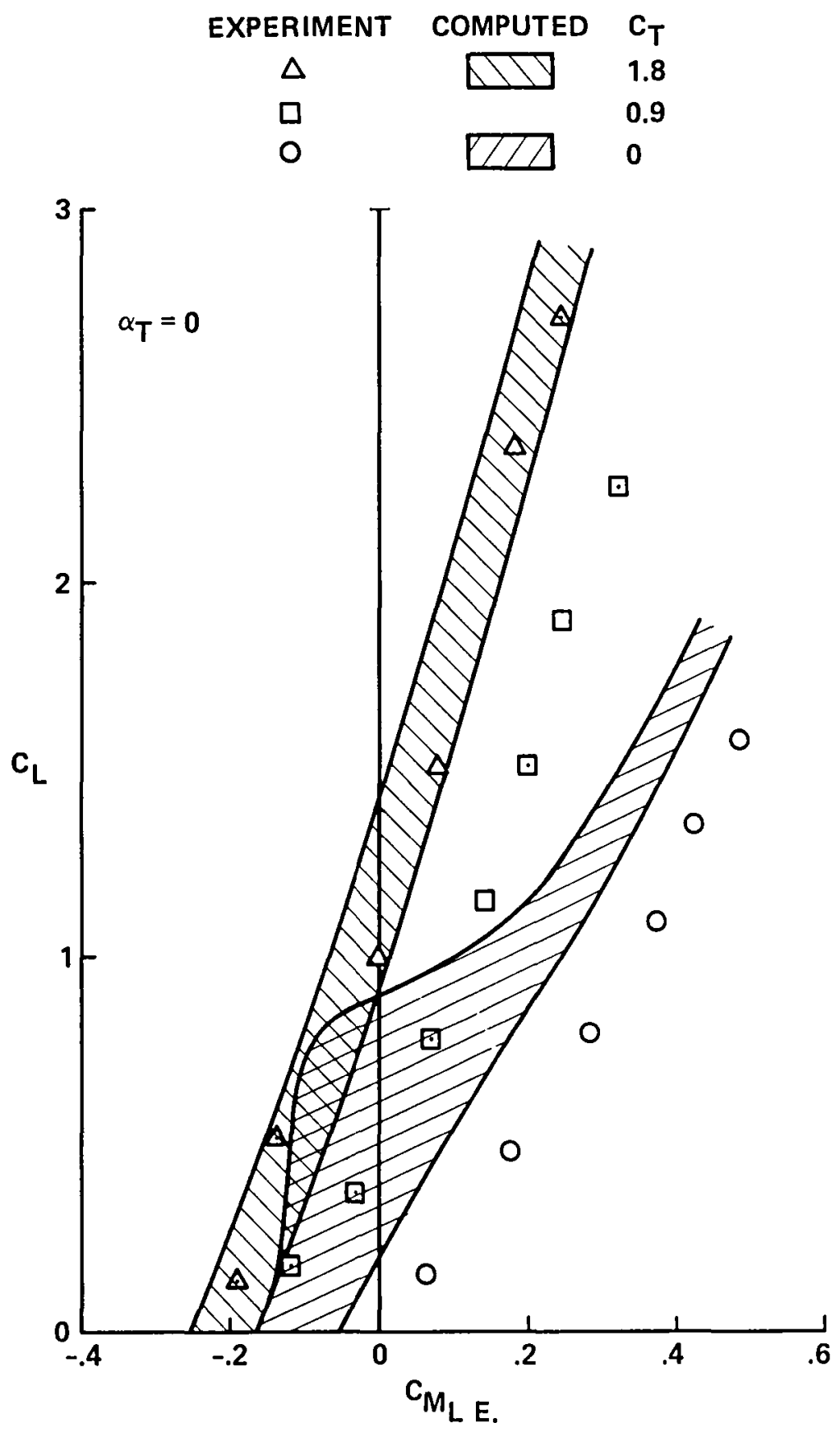

Figure 15.- Pitching moment diagram for the V/STOL fighter model. 


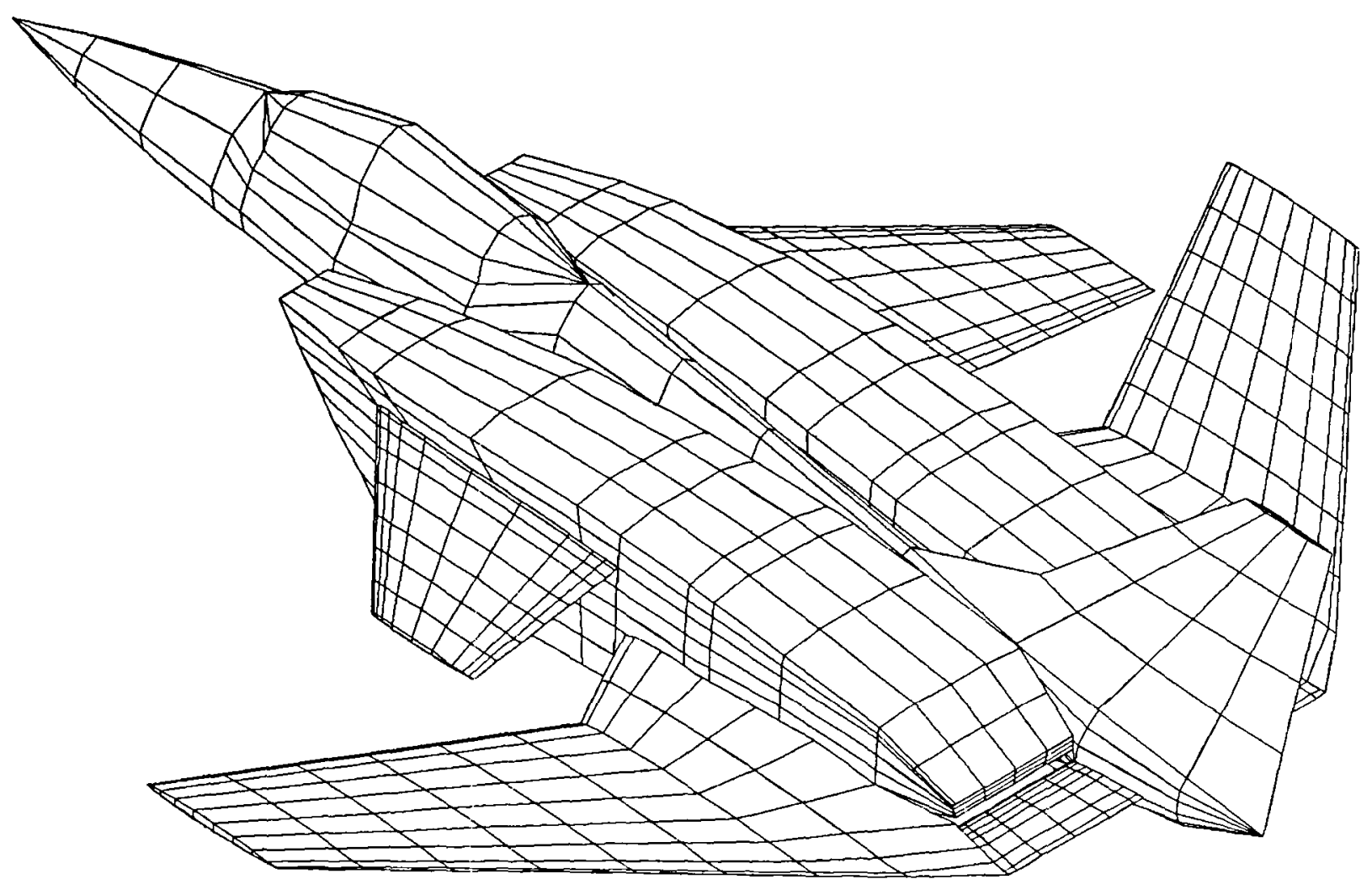

Eigure 16.- Panel model of the forward-swept-wing V/STOL fighter. 

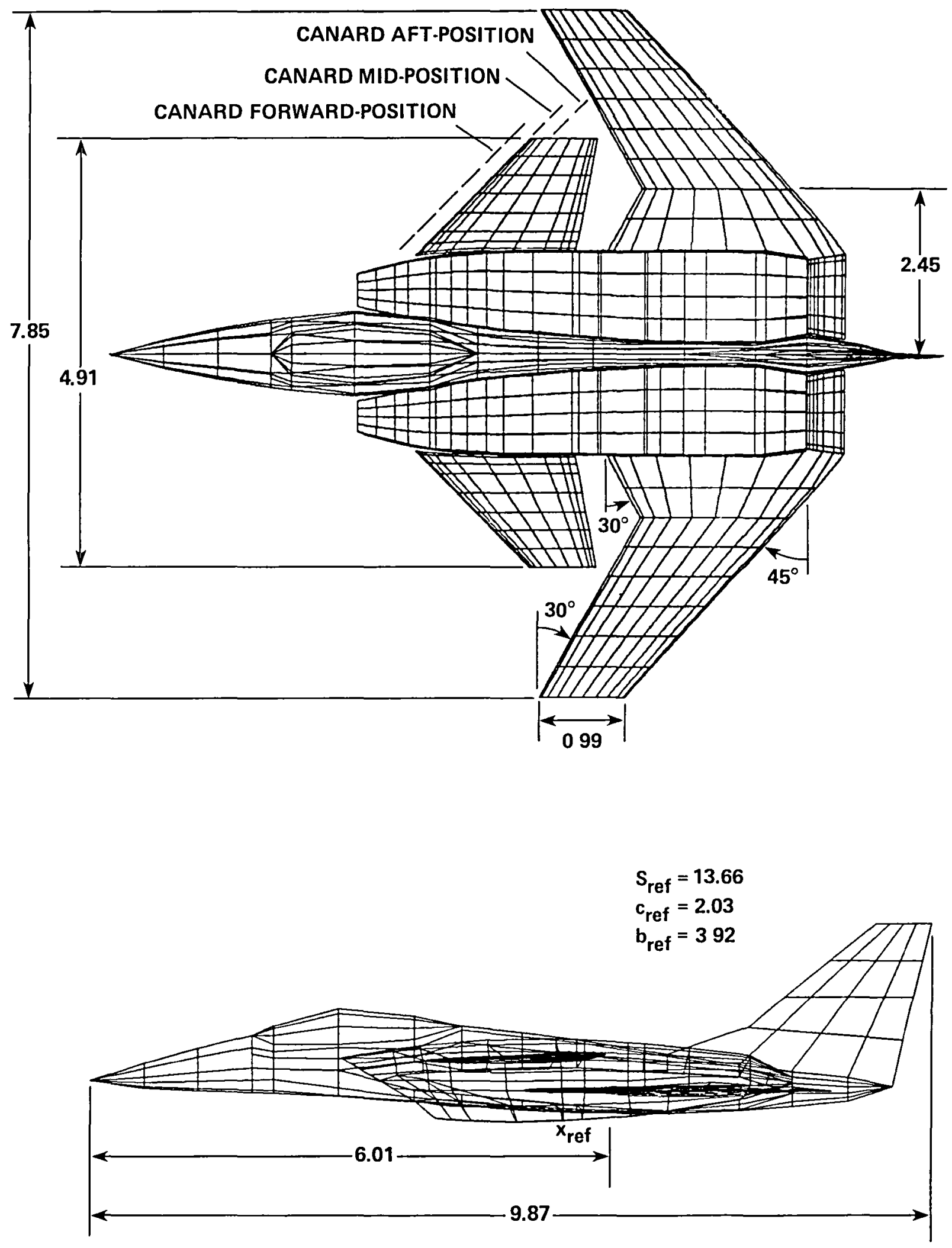

Figure 17.- Geometrical detalls of the forward-swept-wing model. 


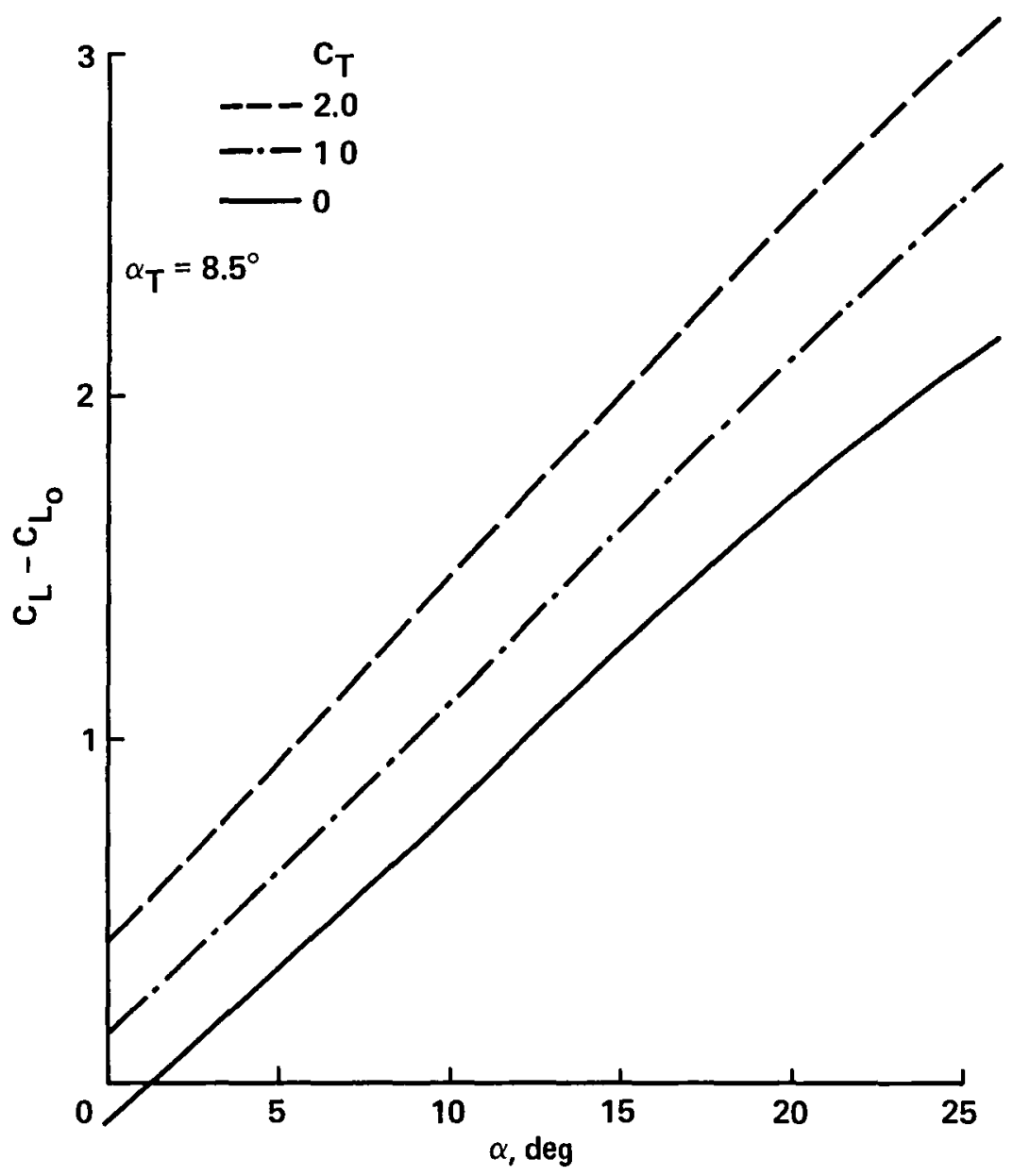

Figure 18.- Prediction of lift coefficients for the forward-swept-wing model.

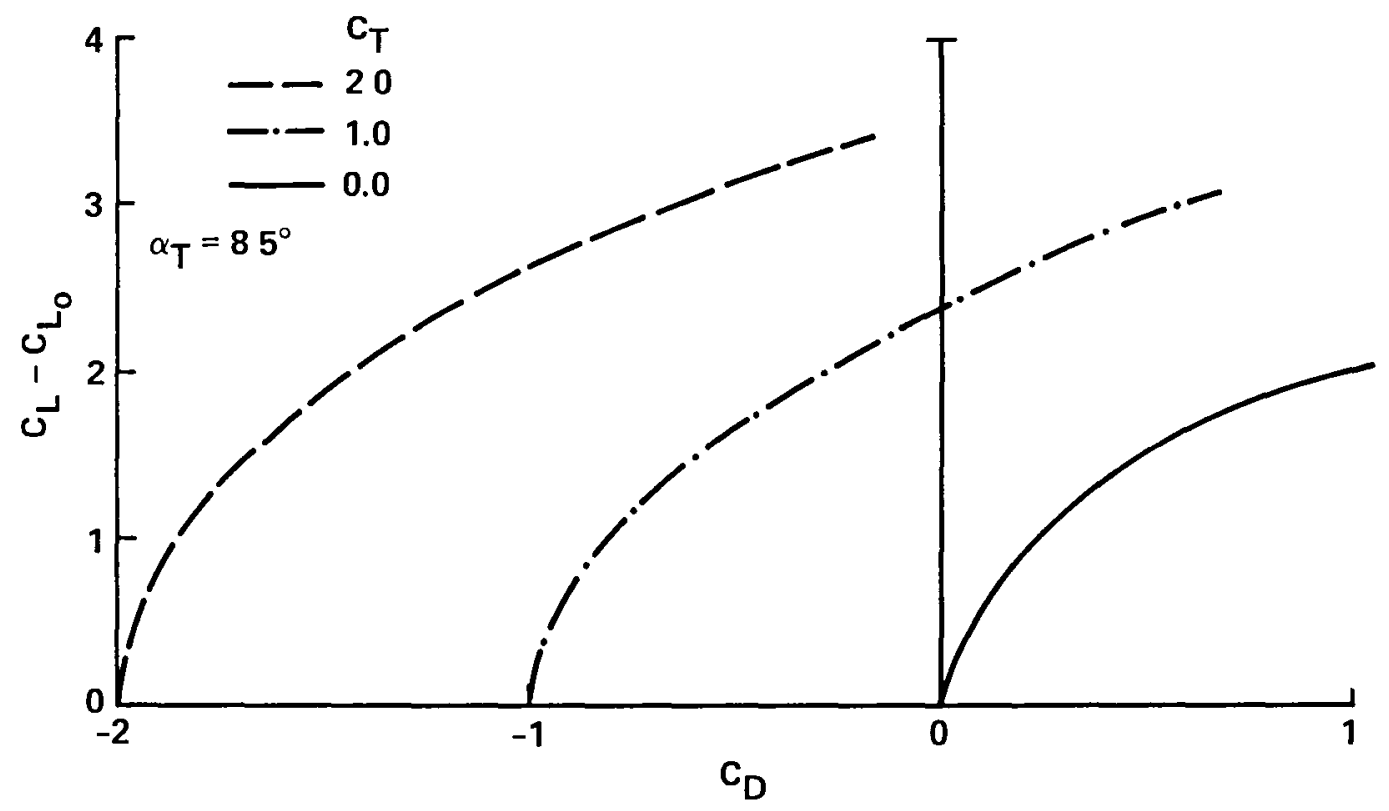

Figure 19.- Lift/drag polars for the forward-swept-wing model. 


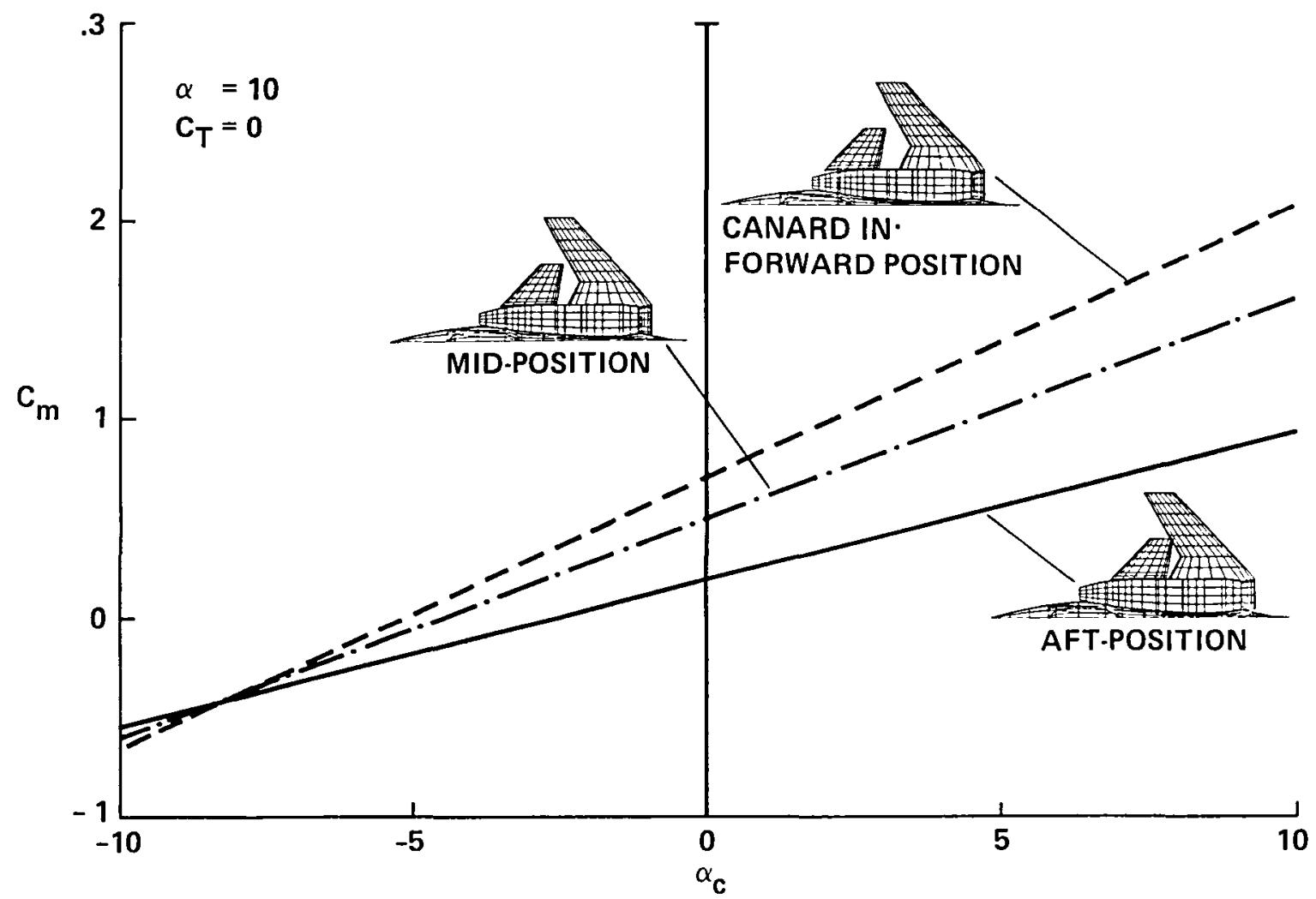

Figure 20.- Effect of canard position on pitching moment.

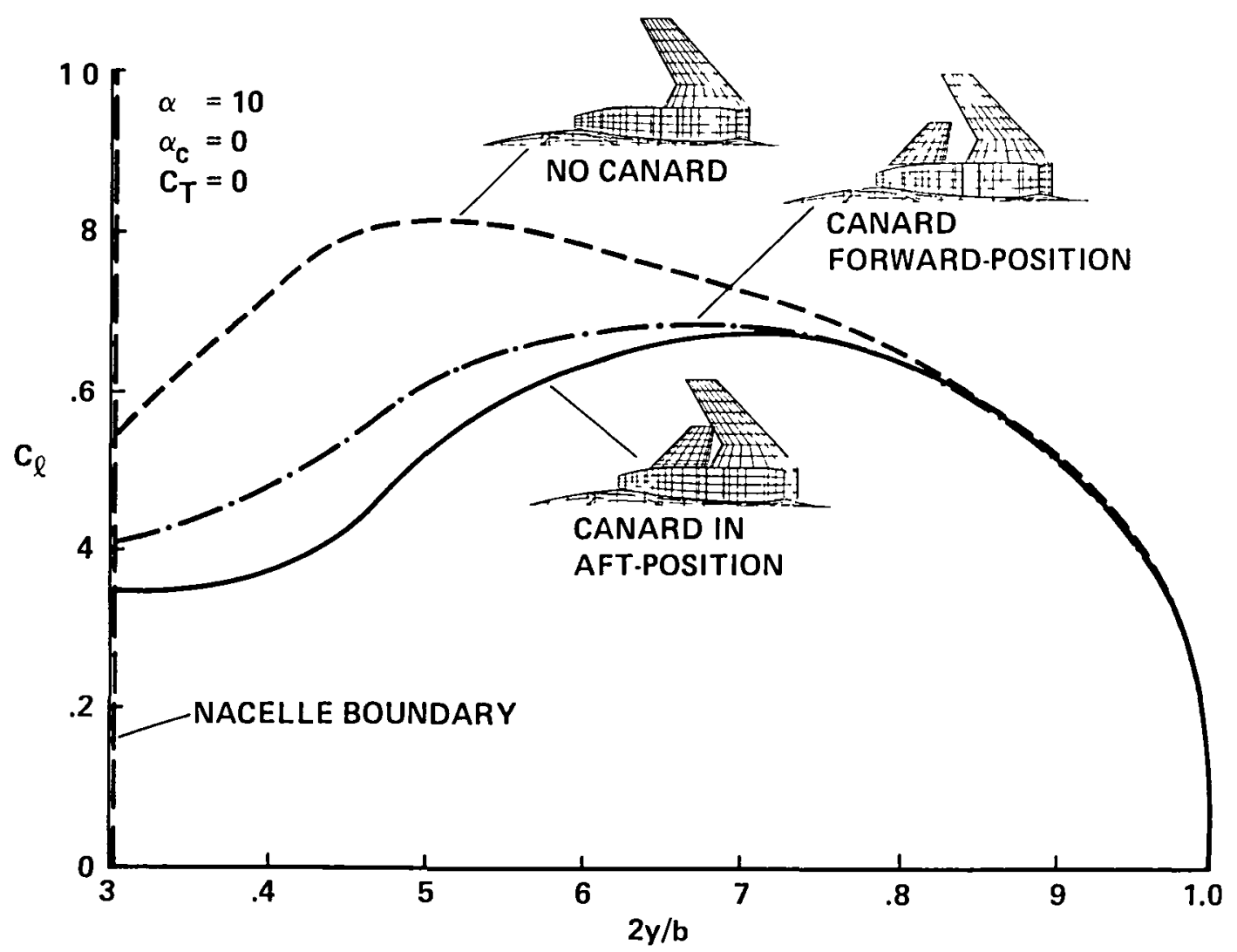

Figure 21.- Effect of canard position on wing's spanwise loading. 


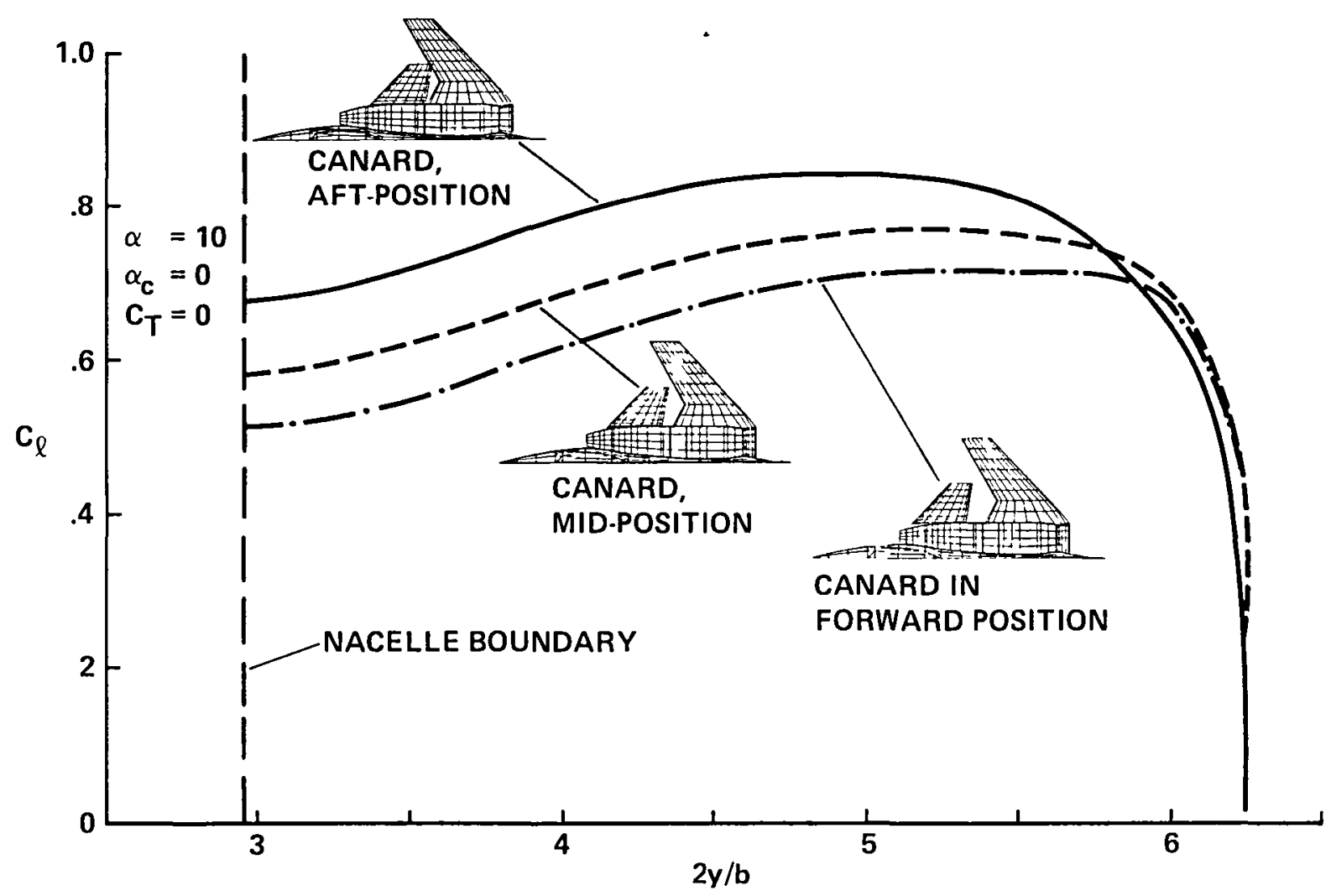

Figure 22.- Effect of canard position on canard's spanwise loading.

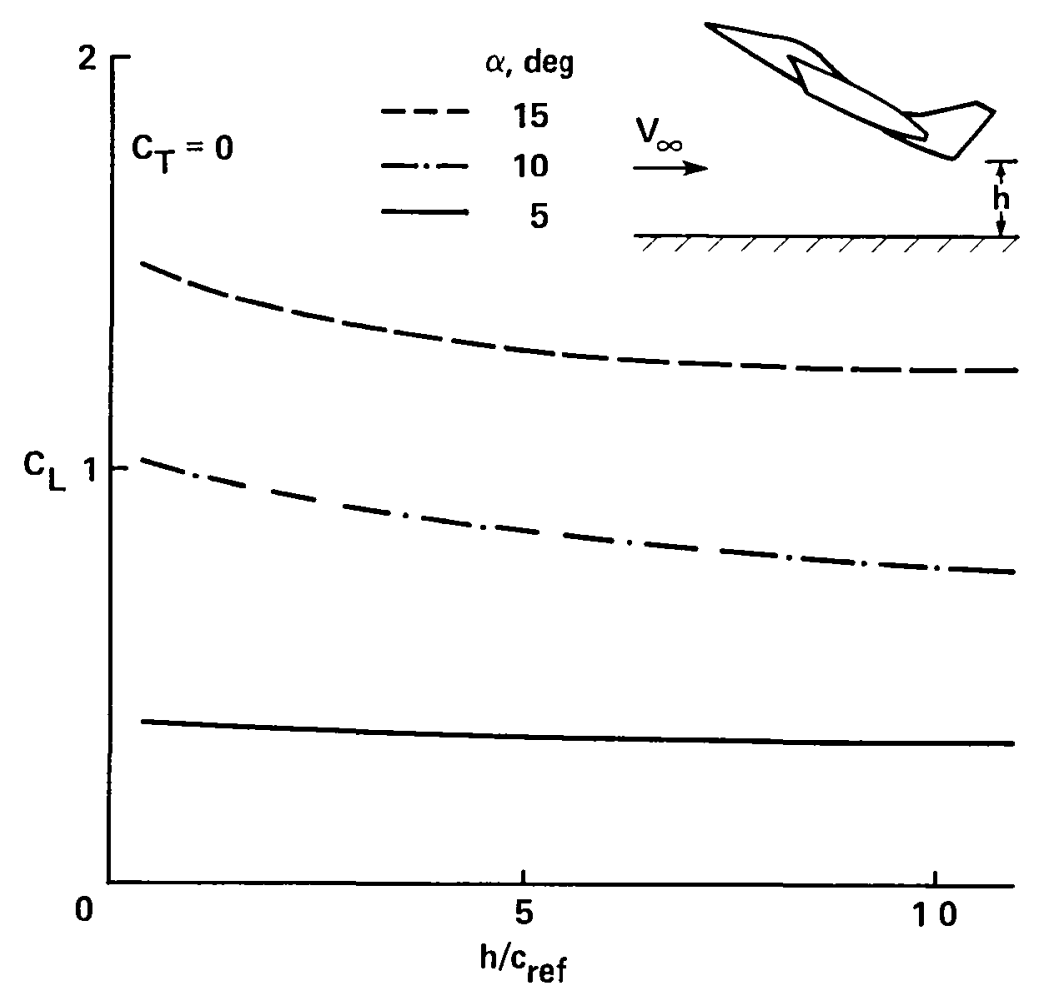

Figure 23.- Effect of ground proximity on lift. 


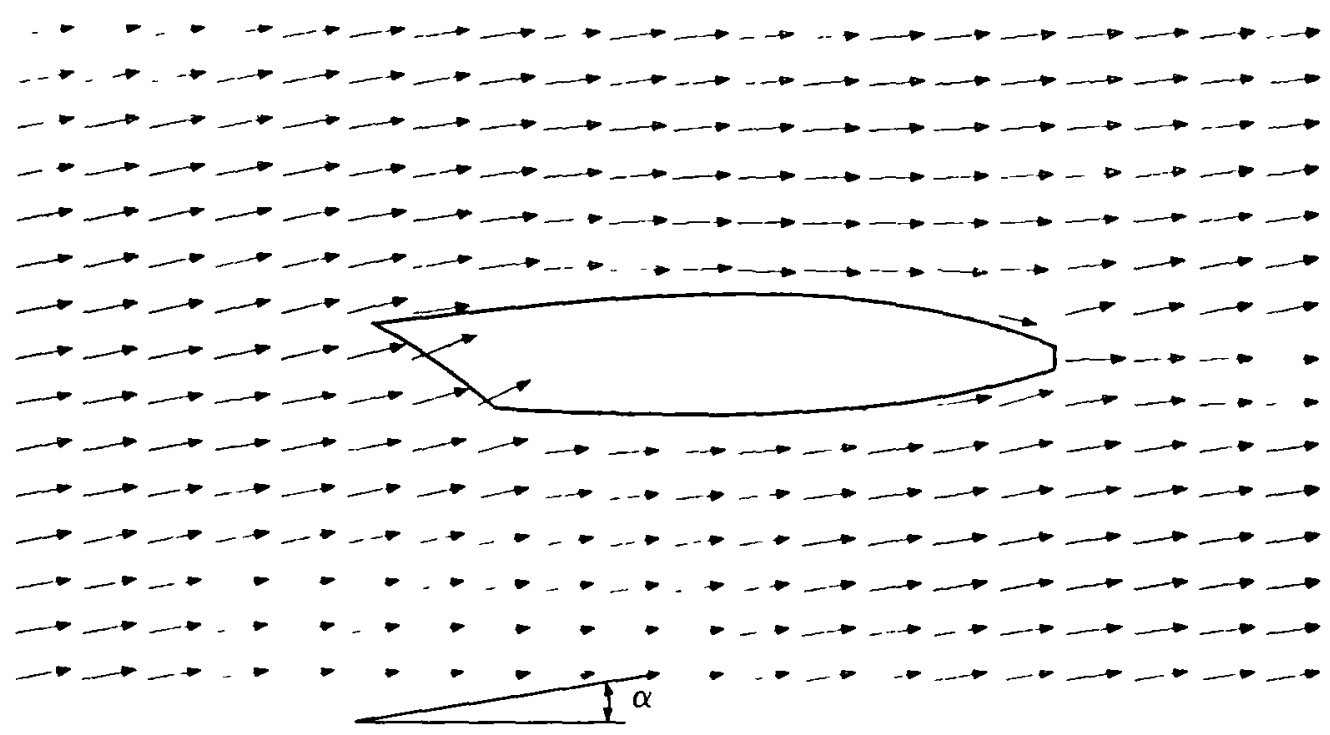

Figure 24.- Numerical-flow visualization of the external flow at the nacelle's midsection.

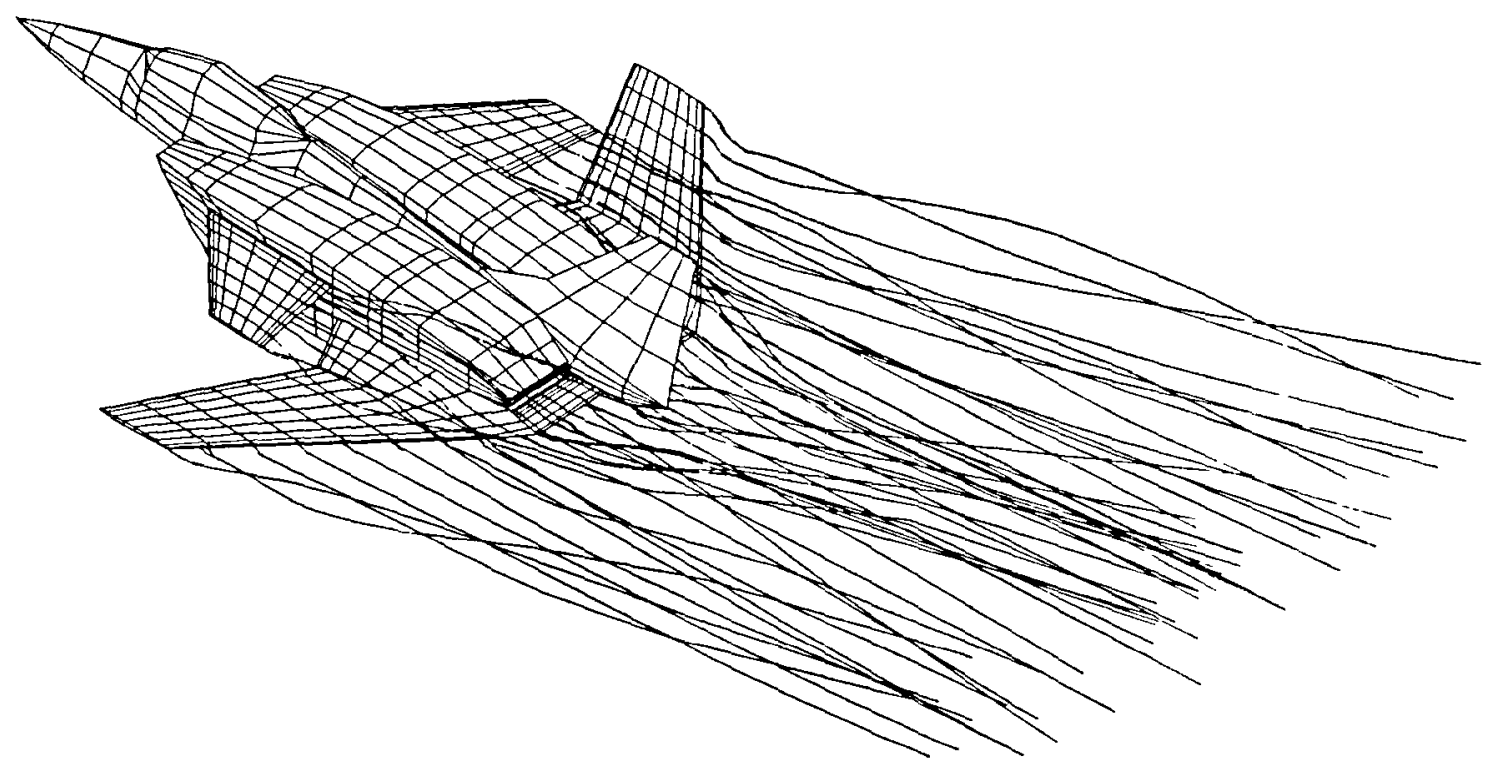

Figure 25.- Numerical-flow visualization of aircraft wakes. 


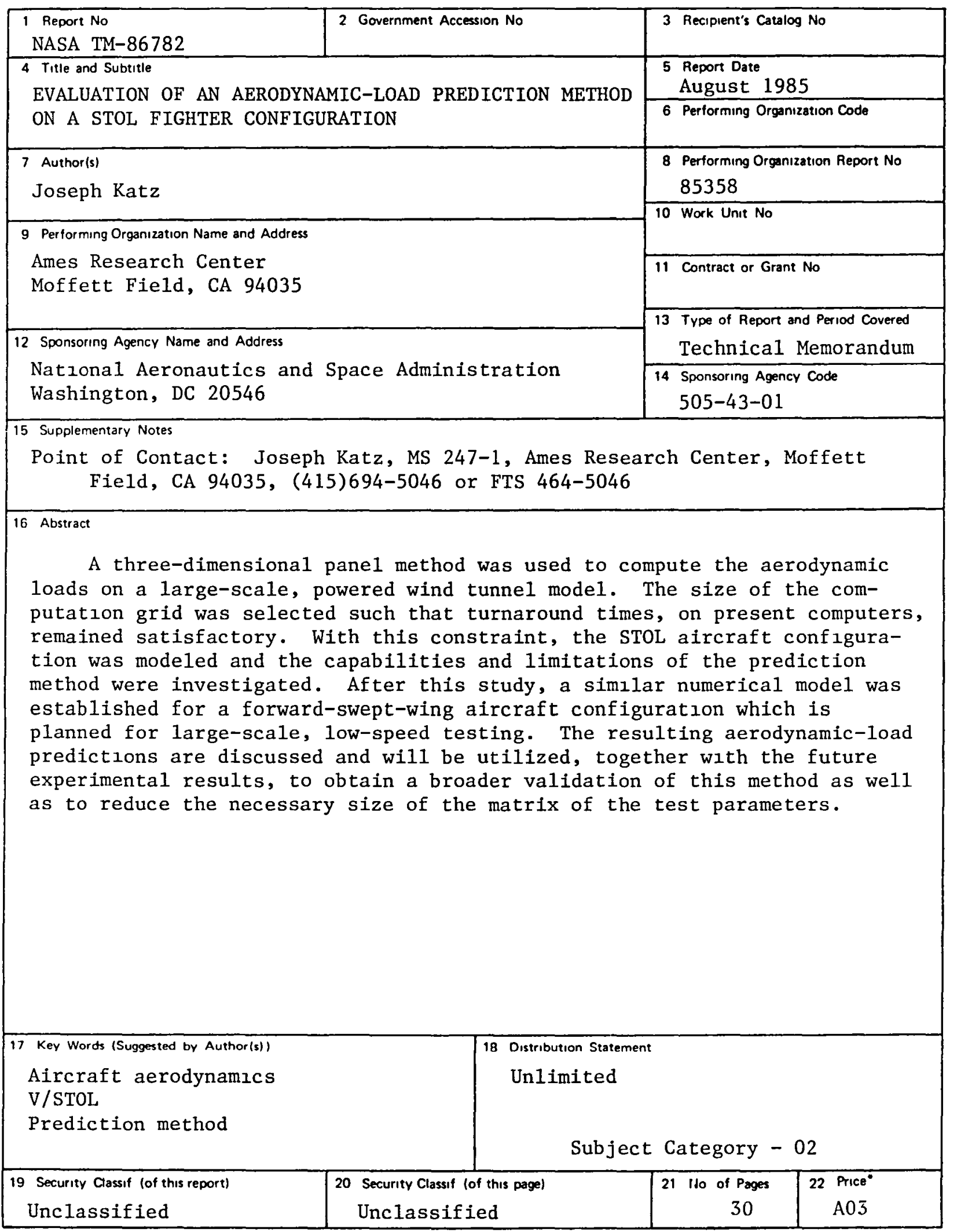

- For sale by the National Technical Information Service, Springfield, Virginia 22161 
End of Document 